\title{
Uncertainty Quantification in DIC with Kriging Regression
}

\author{
Dezhi Wang ${ }^{1}$, F.A. DiazDelaO ${ }^{1,2}$, Weizhuo Wang ${ }^{3}$, Xiaoshan Lin ${ }^{4}$, \\ Eann A. Patterson ${ }^{4}$ and John E. Mottershead ${ }^{1,2}$ \\ ${ }^{1}$ Centre for Engineering Dynamics, University of Liverpool, UK. \\ ${ }^{2}$ Institute for Risk and Uncertainty, University of Liverpool, UK. \\ ${ }^{3}$ School of Engineering, Manchester Metropolitan University, UK. \\ ${ }^{4}$ Centre for Materials and Structures, University of Liverpool, UK \\ j.e.mottershead@liverpool.ac.uk
}

\begin{abstract}
A Kriging regression model is developed as a post-processing technique for the treatment of measurement uncertainty in classical subset-based Digital Image Correlation (DIC). Regression is achieved by regularising the sample-point correlation matrix using a local, subset-based, assessment of the measurement error with assumed statistical normality and based on the Sum of Squared Differences (SSD) criterion. This leads to a Kriging-regression model in the form of a Gaussian process representing uncertainty on the Kriging estimate of the measured displacement field. The method is demonstrated using numerical and experimental examples. Kriging estimates of displacement fields are shown to be in excellent agreement with 'true' values for the numerical cases and in the experimental example uncertainty quantification is carried out using the Gaussian random process that forms part of the Kriging model. The root mean square error (RMSE) on the estimated displacements is produced and standard deviations on local strain estimates are determined. Significant improvement is observed on strain results produced using a commonly used pointwise least squares algorithm (PLS).
\end{abstract}

Keywords: Digital Image Correlation, Measurement Error, Kriging Regression, Uncertainty Quantification.

\section{Introduction}

Digital Image Correlation (DIC) is a well-developed and extensively applied technique in experimental mechanics while subset-based DIC is probably the most commonly used approach because of its simplicity [1]. Inaccuracy, typically caused by camera noise, illumination variability, grey-scale interpolation and other sources, will always be present regardless of the level of precision of the DIC measurement. The resulting error in the measured data affects the accuracy of strain estimates [2] 
based on displacement data. A common way of dealing with this problem is to apply local smoothing [3,4] or low-pass filtering [5], which has the advantage of simplicity but is subject to ad-hoc choice of order and parameterisation, possibly leading only to a local optimum. In the present study a global improvement in measurement accuracy is sought by post-processing with a Kriging model that incorporates knowledge of error estimates determined from classical subset-based DIC. Specifically, the measured data is regressed by utilizing an estimate of the measurement error built into the leading diagonal of the Kriging correlation matrix [6-9].

Originally developed in geology [10], Kriging is also widely used in the fields of spatial analysis and computer experiments. The complete history and extensive overview on the development of Kriging can be found in the work of Cressie [11, 12]. As opposed to a piecewise-polynomial spline that optimizes smoothness of the fitted data, Kriging is a method that gives the best linear unbiased prediction (BLUP) [12] of intermediate values. Also Kriging can be interpreted from a Bayesian framework $[12,13]$ i.e. the interpolated values are modelled by a Gaussian process governed by prior covariance. The idea of introducing a regularization factor for the treatment of biased estimates in multiple regression can be traced back to the work of Hoerl and Kennard [6] in 1970, who termed the technique 'ridge regression'. Forrester et al. [7] applied the same approach to the design and analysis of 'noisy' computer experiments in the field of computer simulations in order to filter out numerical noise. The introduction of a single regularization factor is a global approach for the treatment of uniform uncertainty across the domain of interest.

Jouke et al. [8] extended the error estimate from the global to the local domain and proposed a local error estimate technique in particle image velocimetry (PIV) by using an uncertainty model based on peak ratios [14] in the cross-correlation map. This technique is not transferrable to DIC because of significant differences in the cross-correlation map of DIC data compared with PIV. Also DIC algorithms are usually based on the Sum of Squared Difference (SSD) criterion and Newton iteration. Sutton et al. $[1,15]$ derived an estimate of displacement error due to the presence of Gaussian image noise, which is a function of the standard deviation of Gaussian noise and the sum of squared intensity gradients [16]. By considering the various error sources existing in the experiments, a more general form of error estimate was derived to approximate DIC error bounds as a function of the SSD residual and the inverse of Hessian matrix [17, 18]. It is advantageous that the error estimate can be determined in the DIC process simultaneously with the displacement data without increasing the computational cost.

A Kriging full-field DIC algorithm was developed by the present authors [19] who considered the measurement uncertainty to be independent and identically distributed across the entire Region of Interest (RoI). Whilst this approach offers excellent error reduction properties, the resulting Gaussian-process estimate is limited by the assumption of measurement error that, within the RoI, remains the same from location to location. This limitation is addressed in the present article whereby a local error 
estimate based on the inverse Hessian matrix and the residual of the SSD criterion is incorporated in Kriging regression. Whereas in [19] the Kriging model was used as a shape function in the full-field DIC Newton iteration, in the present study Kriging regression is used as a post-processing technique to improve the accuracy of classical subset-based DIC measurement by including a local error estimate determined subsetby-subset. Numerical and experimental examples are used to test the performance of the proposed approach. One of the advantages of Kriging is that it provides not only a best linear unbiased prediction of the measurement, but also a Gaussian random process that delivers uncertainty quantification (UQ) on the prediction itself. Results show that Kriging regression with local error estimation is able to reduce the effect of measurement errors and improve the accuracy of the estimated displacement field rather than just smoothing it. In an experimental example, the RMSE on the estimated displacement field and the standard deviations on locally estimated strains are presented. Post-processing with the Kriging model leads to a significant improvement in strain results obtained using an extensively used local linear fitting algorithm with a strain calculation window of various sizes [4]. The strain results determined directly from the gradients of the Kriging displacement field are also presented for comparison.

\section{Uncertainty in Subset-based DIC}

In order to obtain a mathematical expression for the local error estimate to be incorporated in the Kriging regression, a generic analysis for the measurement uncertainty of subset-based DIC is introduced first. We begin with the assumption of deformation continuity of a solid object and for reasons of simplicity a 2-dimensional case is considered. A point at coordinate $\mathbf{x}_{c}:\left(x_{c}, y_{c}\right)$ defines the central node of a reference grey-level image $f(x, y)$, which for convenience takes the form of a square subset consisting of $N \times N$ pixels. The central-node coordinate of the smoothly deformed grey-level image $g(\tilde{x}, \tilde{y})$ is given by $\tilde{\mathbf{x}}_{c}:\left(\tilde{x}_{c}, \tilde{y}_{c}\right)$.

The grey-level images $\bar{f}(x, y)$ and $\bar{g}(\tilde{x}, \tilde{y})$ consist of the true images, $f(x, y)$ and $g(\tilde{x}, \tilde{y})$, plus the grey-intensity error defined by $\zeta_{f}(x, y)$ and $\zeta_{g}(\tilde{x}, \tilde{y})$ respectively,

$$
\begin{aligned}
& \bar{f}(x, y)=f(x, y)+\zeta_{f}(x, y) \\
& \bar{g}(\tilde{x}, \tilde{y})=g(\tilde{x}, \tilde{y})+\zeta_{g}(\tilde{x}, \tilde{y})
\end{aligned}
$$

where $\zeta_{f}(x, y), \zeta_{g}(\tilde{x}, \tilde{y})$, to be estimated experimentally, are assumed Gaussian $\mathcal{N}\left(0, \sigma_{\zeta}^{2}\right)$, independent and identically distributed across the subset.

In addition, there is measurement error in the difference between the deformed- and reference-image coordinates denoted by $\boldsymbol{\varepsilon}_{e}$. Then the arbitrarily measured coordinate $\tilde{\mathbf{x}}_{s}:\left(\tilde{x}_{s}, \tilde{y}_{s}\right)$ may be written as, 


$$
\tilde{\mathbf{x}}_{s}=\boldsymbol{\tau}_{s}+\boldsymbol{\varepsilon}_{e}
$$

and $\boldsymbol{\tau}_{s}$ denotes the true coordinate of the deformed image.

The true coordinate, and therefore the displacement error is unknown. However, it is assumed that the error across each subset can be modelled using a shape function. This leads to the formulation of weighting terms that account for different error levels in different regions of the full image. Full detailed derivation of the weighting terms is given in Section 3.2 and Appendix 1.

The Kriging regression with local error estimate, described in the following section, is applied in the form of a non-parametric regression model and by including certain weighting terms, uncertainty in different parts of the full image may be represented probabilistically to develop an estimate of the true displacement field. Numerical and experimental examples show that the proposed approach is able to improve the measurement results of the classical subset-based method and outperforms the global Kriging DIC method [19].

\section{Kriging Regression with Local Error Estimate}

Typically, DIC data are not measured with perfect accuracy, but are subject to measurement noise and imprecision [6, 7, 20-22], which might be reduced by prefiltering [5, 23, 24]. However, in this study the Kriging regression approach accounts for measurement error in an overall way by regularizing the diagonal elements of the Kriging correlation matrix R. This means that the training points (or sample points) are not reproduced exactly but allow for error in the measured DIC image, thereby enabling the determination of an optimised displacement field represented by the Kriging model that represents the true displacement in the sense of a best linear unbiased prediction.

The method applied in this study is Kriging regression, also known as 'Universal Kriging' or 'Kriging with External Drift' [25]. Specifically in this study, the true displacement field $w(x, y)$ is modelled using Kriging as a realisation in the form of a random function $\hat{w}(x, y)$, which combines a deterministic regression model with a zero-mean stochastic field used to fit the residuals [26, 27] as,

$$
\hat{w}(x, y)=\sum_{\ell=1}^{m} c_{\ell}(x, y) \beta_{\ell}+Z(x, y)
$$

where $c_{\ell}(x, y), \ell=1, \ldots, m$, are regression functions, $\beta_{\ell}$ denotes the $\ell^{\text {th }}$ regression parameter and $Z(x, y)$ is a Gaussian stochastic field with zero mean and covariance between two arbitrary sample points $j$ and $k$, assumed to take the form,

$$
\operatorname{cov}\left(Z\left(\mathbf{x}_{j}\right), Z\left(\mathbf{x}_{k}\right)\right)=\sigma^{2} r_{j k}\left(\mathbf{x}_{j}, \mathbf{x}_{k}, \vartheta_{x}, \vartheta_{y}, \xi\right) ; \quad \mathbf{x}_{j}=\left(x_{j}, y_{j}\right)^{\mathrm{T}} ; \quad \mathbf{x}_{k}=\left(x_{k}, y_{k}\right)^{\mathrm{T}}
$$


where $r_{j k}\left(\mathbf{x}_{j}, \mathbf{x}_{k}, \vartheta_{x}, \vartheta_{y}\right)=\operatorname{corr}\left(Z\left(\mathbf{x}_{j}\right), Z\left(\mathbf{x}_{k}\right)\right)$ is determined by the proximity of points $j$ and $k$. The correlation parameters $\vartheta_{x}, \vartheta_{y}, \xi$ and field variance $\sigma^{2}$ are determined by an optimisation procedure described in the sequel.

The regression parameters $\beta_{\ell}$ may be estimated from the sample by using the generalized least squares (GLS) method $[12,28]$. Denoting $\mathbf{w}_{0}=\left[w_{1}, \cdots, w_{n}\right]^{\mathrm{T}}$ as the vector of displacements calculated by the subset-based DIC for a set of sample points $\left(x_{j}, y_{j}\right), j=1,2, \ldots, n$, the estimated regression parameters $\hat{\boldsymbol{\beta}}$ are then expressed as [28],

$$
\hat{\boldsymbol{\beta}}=\left(\mathbf{C}^{\mathrm{T}}\left(\mathbf{R}\left(\vartheta_{x}, \vartheta_{y}, \xi\right)\right)^{-1} \mathbf{C}\right)^{-1} \mathbf{C}^{\mathrm{T}}\left(\mathbf{R}\left(\vartheta_{x}, \vartheta_{y}, \xi\right)\right)^{-1} \mathbf{w}_{0}
$$

Then, by minimising the mean-square prediction error under an unbiasedness constraint [28], the Kriging model at an arbitrarily chosen point $\mathbf{x}_{p}=\left(x_{p}, y_{p}\right)$ is obtained as,

$$
\hat{w}\left(\mathbf{x}_{p}\right) \sim \mathcal{N}\left(\bar{w}\left(\mathbf{x}_{p}, \vartheta_{x}, \vartheta_{y}, \xi\right), \sigma^{2} S\left(\mathbf{x}_{p}, \vartheta_{x}, \vartheta_{y}, \xi\right)\right)
$$

where,

$$
\bar{w}\left(\mathbf{x}_{p}\right)=\mathbf{c}^{\mathrm{T}}\left(\mathbf{x}_{p}\right) \hat{\boldsymbol{\beta}}+\mathbf{r}^{\mathrm{T}}\left(\mathbf{x}_{p}, \vartheta_{x}, \vartheta_{y}\right)\left(\mathbf{R}\left(\vartheta_{x}, \vartheta_{y}, \xi\right)\right)^{-1}\left(\mathbf{w}_{0}-\mathbf{C} \hat{\boldsymbol{\beta}}\right)
$$

is the Best Linear Unbiased Prediction (BLUP) with the variance given by,

$$
\begin{aligned}
& \hat{\sigma}^{2} S\left(\mathbf{x}_{p}\right)=\hat{\sigma}^{2}\left(1-\mathbf{r}^{\mathrm{T}}\left(\mathbf{x}_{p}\right) \mathbf{R}^{-1} \mathbf{r}\left(\mathbf{x}_{p}\right)+\left(\mathbf{c}^{\mathrm{T}}\left(\mathbf{x}_{p}\right)-\mathbf{r}^{\mathrm{T}}\left(\mathbf{x}_{p}\right) \mathbf{R}^{-1} \mathbf{C}\right)\right. \\
&\left.\times\left(\mathbf{C}^{\mathrm{T}} \mathbf{R}^{-1} \mathbf{C}\right)^{-1}\left(\mathbf{c}^{\mathrm{T}}\left(\mathbf{x}_{p}\right)-\mathbf{r}^{\mathrm{T}}\left(\mathbf{x}_{p}\right) \mathbf{R}^{-1} \mathbf{C}\right)^{\mathrm{T}}\right)
\end{aligned}
$$

Covariance terms may be expressed as,

$$
\begin{gathered}
\hat{\sigma}^{2} S\left(\mathbf{x}_{p}, \mathbf{x}_{q}\right)=\hat{\sigma}^{2}\left(r_{p q}\left(\mathbf{x}_{p}, \mathbf{x}_{q}\right)-\mathbf{r}^{\mathrm{T}}\left(\mathbf{x}_{p}\right) \mathbf{R}^{-1} \mathbf{r}\left(\mathbf{x}_{q}\right)+\left(\mathbf{c}^{\mathrm{T}}\left(\mathbf{x}_{p}\right)-\mathbf{r}^{\mathrm{T}}\left(\mathbf{x}_{p}\right) \mathbf{R}^{-1} \mathbf{C}\right)\right. \\
\left.\times\left(\mathbf{C}^{\mathrm{T}} \mathbf{R}^{-1} \mathbf{C}\right)^{-1}\left(\mathbf{c}^{\mathrm{T}}\left(\mathbf{x}_{q}\right)-\mathbf{r}^{\mathrm{T}}\left(\mathbf{x}_{q}\right) \mathbf{R}^{-1} \mathbf{C}\right)^{\mathrm{T}}\right)
\end{gathered}
$$

where $\mathbf{x}_{p}, \mathbf{x}_{q}$ may be either sampled or unsampled points and $(\hat{\bullet})$ denotes an estimate.

In the above expressions, $\mathbf{R}$ is the matrix of sample-point displacement correlation functions with terms $r_{j k}\left(\mathbf{x}_{j}, \mathbf{x}_{k}, \vartheta_{x}, \vartheta_{y}, \xi\right)$ described above; $\mathbf{r}\left(\mathbf{x}_{p(q)}, \vartheta_{x}, \vartheta_{y}\right)$ is the vector of displacement correlation functions between an arbitrarily chosen location $\left(\mathbf{x}_{p(q)}\right)$ and each of the sample points $\left(\mathbf{x}_{j}\right), j=1,2, \ldots, n$; and $r_{p q}\left(\mathbf{x}_{p}, \mathbf{x}_{q}, \vartheta_{x}, \vartheta_{y}\right)$ denotes the 
correlation between two arbitrarily chosen points $\left(\mathbf{x}_{p}, \mathbf{x}_{q}\right) . \mathbf{C}$ is a matrix consisting of regression functions evaluated at the sample points, $C_{\ell j}=c_{\ell}\left(\mathbf{x}_{j}\right)$; and $\mathbf{c}\left(\mathbf{x}_{p(q)}\right)$ is the vector of regression functions for an arbitrary location $\mathbf{x}_{p(q)}$, i.e. $c_{\ell}=c_{\ell}\left(\mathbf{x}_{p(q)}\right)$.

The correlation functions are generally assumed to be exponential, also called Gaussian [9], and expressed in the form,

$$
r_{p q}\left(\mathbf{x}_{p}, \mathbf{x}_{q}, \vartheta_{x}, \vartheta_{y}\right)=\exp \left(-\vartheta_{x}\left(x_{p}-x_{q}\right)^{2}-\vartheta_{y}\left(y_{p}-y_{q}\right)^{2}\right)
$$

The choice of this correlation function relies on the assumption that the response surface inferred by Kriging regression is smooth. It is seen from equation (11) that points close to each other have a higher correlation than those that are far away. The terms $\vartheta_{x}$ and $\vartheta_{y}$ determine how far apart both $x_{p}$ and $x_{q}$ and $y_{p}$ and $y_{q}$ need to be before differences in the estimate given by equation (11) become significant.

When measurement error is considered the diagonal elements of the correlation matrix $\mathbf{R}$ in the Kriging formula should be adjusted by the introduction of an error term (multiplicatively in the present work). This allows for regression instead of exact interpolation of the data samples. According to the correlation function (11), all the diagonal elements of matrix $\mathbf{R}$ are unity for Kriging interpolation which means that the Kriging model passes through all the samples exactly. In contrast, measurement error is taken into account by regularizing the diagonal elements of the correlation matrix $\mathbf{R}$, which allows regression of the Kriging model on the data samples. This modified formulation is known as Kriging regression [21] and introduces the additional parameter $\xi$.

\subsection{Global error estimate}

If the error is assumed independent and identically distributed across the entire region of interest then an unknown constant factor $\xi$ may be applied [7, 8, 19] to modify the diagonal elements of the correlation matrix $\mathbf{R}$, which then differ from equation (11) and are given by,

$$
r_{j j}=\exp (\xi) ; \quad j=1,2, \ldots, n
$$

where $n$ denotes the number of sample points.

\subsection{Local error estimate}

In many cases, the error is not constant over the entire domain but may separately be considered independent and identically distributed over a subset of the reference image. An estimate of displacement error for each subset due to the presence of Gaussian image noise was derived $[1,15]$ as a function of the standard deviation of Gaussian noise and the sum of squared intensity gradients [16]. In this study, a more 
general form of error estimate is derived to approximate the DIC error bound for each subset as a function of the SSD residual and the inverse of Hessian matrix [17, 18]. The multiple error sources in DIC measurement are thereby included in a general way. It is shown in Appendix 1 that this general form of error estimate for each subset may be expressed as,

$$
\begin{aligned}
& m_{x j}=\frac{C_{S S D j}}{N^{2}} \cdot\left(\mathbf{H}^{-1}\right)_{11} \\
& m_{y j}=\frac{C_{S S D j}}{N^{2}} \cdot\left(\mathbf{H}^{-1}\right)_{77}
\end{aligned}
$$

where $m_{x j}$ and $m_{y j}$ are approximations to the error variances associated with $x$ - and $y$ direction displacements for the $j^{\text {th }}$ subset of $N \times N$ pixels, having a single sample point at its centre. $C_{S S D j}$ denotes the SSD residual for the $j^{\text {th }}$ subset. $\mathbf{H}$ is the calculated Hessian matrix while subscripts 11 and 77 indicate the diagonal elements of $\mathbf{H}^{-1}$ that corresponds to the $x$ - and $y$-direction displacements of the subset centre point.

If the different error for each subset is taken into account, equation (12) may then be expressed in modified form,

$$
r_{j j}=\exp \left(\xi\left(\frac{-\vartheta_{x} m_{x j}-\vartheta_{y} m_{y j}}{\sqrt{m_{x 1}^{2}+m_{y 1}^{2}}}\right)\right)
$$

where the term $\sqrt{m_{x 1}^{2}+m_{y 1}^{2}}$ is a normalising constant.

The derivation of $m_{x j}$ and $m_{y j}$, given in Appendix 1, is achieved under the following conditions on the measurement error:

i. Assumed to be Gaussian with zero mean, independent and identically distributed over a subset of $N \times N$ pixels.

ii. Approximated using shape functions based on a second-order Taylor series expansion (may be first- or higher-order) about a sample point at a subset centre.

iii. Linearised at $N \times N$ pixels to relate field uncertainties to shape function variables.

iv. Pixel grey-intensity variances approximated using the SSD between the deformed and reference images.

3.3 Solution of unknown parameters $\left\{\vartheta_{x}, \vartheta_{y}, \xi, \hat{\sigma}^{2}\right\}$. 
The parameters $\left\{\vartheta_{x}, \vartheta_{y}, \xi\right\}$ determined by maximising the concentrated log likelihood function [7, 9] given by,

$$
\ln L\left(\vartheta_{x}, \vartheta_{y}, \xi\right)=\frac{n}{2} \ln \left(\hat{\sigma}^{2}\right)+\frac{1}{2} \ln \left(\left|\mathbf{R}\left(\vartheta_{x}, \vartheta_{y}, \xi\right)\right|\right)
$$

subject to a constraint on the field variance estimated as [7],

$$
\hat{\sigma}^{2}=\frac{1}{n}\left(\mathbf{w}_{0}-\mathbf{C} \hat{\boldsymbol{\beta}}\right)^{\mathrm{T}} \mathbf{R}^{-1}\left(\vartheta_{x}, \vartheta_{y}, \xi\right)\left(\mathbf{w}_{0}-\mathbf{C} \hat{\boldsymbol{\beta}}\right)
$$

where $L(\bullet)$ denotes the likelihood.

Equation (15) is complex and generally multimodal. Thus, the computation of optimal values for $\vartheta_{x}, \vartheta_{y}$ and $\xi$ usually requires specialised optimisation algorithms and heuristics such as genetic algorithms or gradient-free methods, e.g. the Hooke and Jeeve's algorithm, and the Nelder-Mead simplex algorithm. The latter was employed in this work with a first-order regression function chosen for $\mathbf{c}(x, y)$ [28]. In the case of a large number of sample points $Q$, the computational cost of a conventional maximum likelihood estimate (MLE) could become significant. Fast algorithms are described in [29].

It is necessary in equation (16) to invert the correlation matrix, $\mathbf{R}$, which may be illconditioned and in need of regularisation. Ranjan et al. [30] considered the classical Tikhonov regularisation of the form $(\mathbf{R}+\delta \mathbf{I})$ where $\delta$ is the regularisation parameter or nugget. The optimised parameter $\xi$ in equations (16), (12) and (14) has the same effect, though in a slightly different form. Regularisation introduces the smoothing required in DIC post-processing and results in a regressing, rather than an interpolating random function represented by the Kriging model. It is however necessary to test the condition of matrix $\mathbf{R}$, which can be done by simply determining the condition number. Ranjan et al. [30] developed a formula for the lower bound on $\delta$, given by

$$
\delta_{l b}=\max \left\{\frac{\lambda_{n}\left(\kappa(\mathbf{R})-e^{a}\right)}{\kappa(\mathbf{R})\left(e^{a}-1\right)}, 0\right\}
$$

where $\kappa(\bullet)$ denotes the condition number, $\lambda_{n}$ is the highest eigenvalue of $\mathbf{R}$ and $a \approx 25$ (an empirical term obtained by a large number of numerical simulations). In the examples presented in this work the term $\left(\frac{\lambda_{n}\left(\kappa(\mathbf{R})-e^{a}\right)}{\kappa(\mathbf{R})\left(e^{a}-1\right)}\right)$ was found in every case to be negative, so that the optimised $\xi$ was able to reduce the measurement error without encountering problems in inverting the correlation matrix, $\mathbf{R}$. 


\subsection{Strain Calculation}

Two different methods were used to determine the strains. Firstly, a local fitting technique [4] based on the point-wise least squares algorithm (PLS), within a chosen strain calculation window, was utilized to estimate the strain result from the measured displacement data. Linear coefficients are fitted to approximate the gradients at the centre point of each local strain window. It is known [4] that there might be an insufficient number of valid data points within the strain calculation window at the boundaries or where there are discontinuities. In order to solve this problem, a displacement continuity assumption may be used to extend the displacement field [31] or alternatively the invalid points may be identified and excluded from the local PLS fitting [4]. The latter was applied in this study. It will be demonstrated in the following sections that this sort of boundary effect may be significantly reduced by applying the proposed Kriging regression approach with the local error estimate.

Secondly, the strain results were calculated directly from the gradients of the Kriging displacement model. This second approach is applied in an experimental case study, where estimated strains are compared to those produced by the PLS method. The Kriging gradients are calculated from the Jacobian of the vector of regression functions $\mathbf{c}\left(\mathbf{x}_{p}\right)$ and the vector of correlation functions $\mathbf{r}\left(\mathbf{x}_{p}\right)$ as discussed in [28].

Although the displacement field is Gaussian, the strain field is generally non-Gaussian. A sampling method based on Cholesky decomposition was employed to sample the displacement field from the multivariate Gaussian distribution [32, 33] with the purpose of quantifying the uncertainty on the estimated strain field. Given the Cholesky decomposition of the correlation matrix $\mathbf{S}=\mathbf{\Lambda} \mathbf{\Lambda}^{T}$ (equations (9) and (10)) where $\boldsymbol{\Lambda}$ is a lower triangular matrix, samples of the displacement field, across the region of interest, were generated from,

$$
\hat{\mathbf{w}}=\Lambda \mathbf{n}+\overline{\mathbf{w}}
$$

by sampling from $\mathbf{n} \sim \mathcal{N}(0, \mathbf{I})$. Classical finite differences may then be applied to calculate the gradients and generate the strain results. This requires dense sampling of displacement field (local sampling) to determine the uncertainty on the estimated strains.

\section{Case studies}

Numerical and experimental case studies are presented to illustrate the application of Kriging regression with local error estimation. Two sets of numerical simulation examples were carried out first so that possible errors introduced by the image acquisition system were excluded. In the first numerical example the effectiveness of the proposed Kriging method in displacement estimation is investigated using numerically generated Gaussian speckles with uniform translations, affine deformation and Gaussian image noise. The second numerical example has the same 
numerically generated Gaussian speckles but a uniaxial tensile deformation with a constant strain. Gaussian image noise is employed to verify the performance of proposed Kriging method in strain measurement. In the experimental example, a cantilever beam test is chosen to investigate the performance of the Kriging method in a practical DIC application, since it has a simple analytical solution for comparison with DIC results.

\subsection{Numerical case study 1: verification of the Kriging method for displacement measurement}

A 2-dimensional numerical example is illustrated, using numerically-produced Gaussian speckles [34, 35] (not related directly to the Gaussian process that forms part of the Kriging model) with means uniformly distributed over the RoI. Gaussian speckles are formulated as,

$$
\begin{aligned}
& I(x, y)=\sum_{k=1}^{M} A_{k} \exp \left(-\frac{\left(x-x_{k}\right)^{2}+\left(y-y_{k}\right)^{2}}{\gamma^{2}}\right) \\
& \tilde{I}(x, y)=\sum_{k=1}^{M} A_{k} \exp \left(-\frac{\left(x-x_{k}-u_{0}-u_{x} x-u_{y} y\right)^{2}+\left(y-y_{k}-v_{0}-v_{x} x-v_{y} y\right)^{2}}{\gamma^{2}}\right)
\end{aligned}
$$

where $I$ and $\tilde{I}$ represent the reference- and deformed-image speckle patterns respectively. $M$ denotes the total number of speckle granules, $\gamma$ is the size and $A_{k}$ the peak intensity of each speckle granule and $\left(x_{k}, y_{k}\right)$ represents the position of each speckle uniformly distributed over the RoI. In the present case, 8000 independent and identically distributed speckles are superimposed on an image consisting of 500×500 pixels, each Gaussian speckle having a size of 3 pixels and a peak intensity of 60 . In order to test the proposed method, several deformed images were produced with a combination of (a) rigid-body translation in $x$ and $y$ directions, (b) affine deformation, and (c) Gaussian image noise. The reference and deformed images were digitised using an 8-bit processor. As the grey values of non-integer locations are required in the DIC process, a grey-value interpolation scheme is needed and for reasons of simplicity a bi-cubic spline interpolation scheme was chosen.

The RoI was divided into 100 uniformly distributed sample points, each of which was defined as the centre node of a subset of $41 \times 41$ pixels as shown in Figure 1. Subsetbased DIC, based on a Newton-Raphson scheme, was applied to assess the measurement uncertainty at all the sample points. Since only a linear deformation (affine transformation) was applied, a first-order Taylor-expansion shape function was chosen in order to avoid possible over-fitting. On the basis of this measurement, both Kriging regression with global and local error estimation were employed to regularize the measured data and achieve an estimate of the displacement at each centre node. As the true displacements of the sample points are easily derivable, the residual errors of subset-based DIC, Kriging global and Kriging local methods are shown and may be compared in Figure 2-5. The results shown for Kriging are the mean values of the 
Gaussian Process that represents the Kriging model. It is observed that the residual errors are significantly reduced after the application of Kriging regression with local error estimation. There is very little difference in the residual errors of Figure 2 between the Kriging global and local methods, which is to be expected because uniform translation was applied to the whole RoI. The Kriging global method performs less well in the case of an affine deformation as shown in Figure 3 because the local deformations differ from subset to subset. Both the Kriging global and local methods demonstrate effective reduction of measurement errors due to Gaussian image noise as shown in Figure 4. In Figure 5 under the effect of combined error sources, the Kriging local method considerably out-performs the Kriging global method.

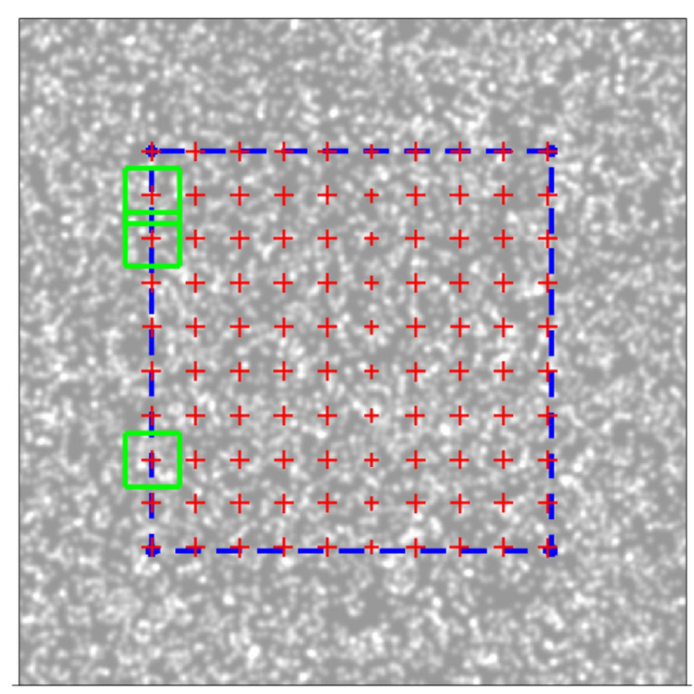

Figure 1 Numerically generated speckles and the distribution of sample points (red crosses) - 3 subsets are shown in green squares

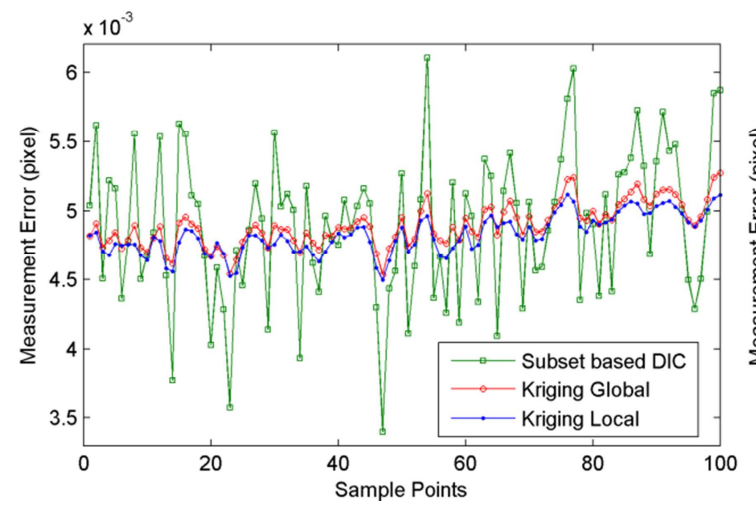

(a) x-direction

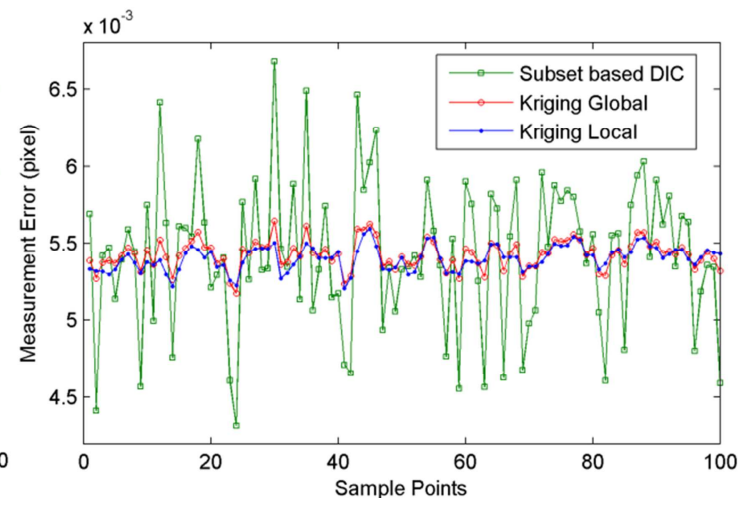

(b) y-direction

Figure 2 Numerical case study 1: residual error comparison for a rigid-body translation $u_{0}=0.2, v_{0}=0.3$ pixels - equation (19). 


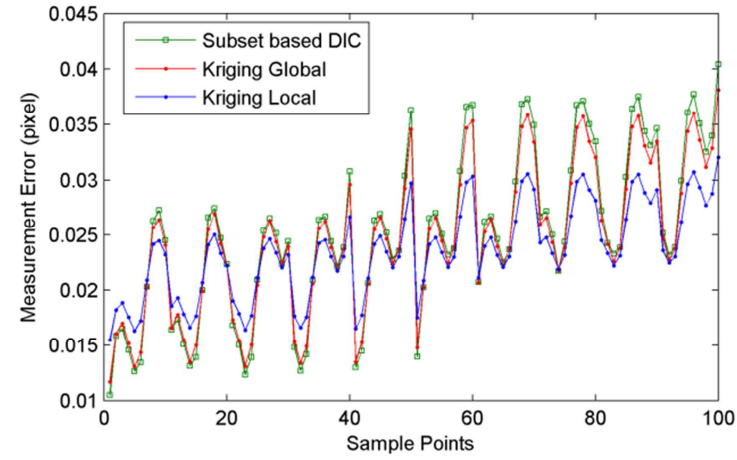

(a) $\mathrm{x}$-direction

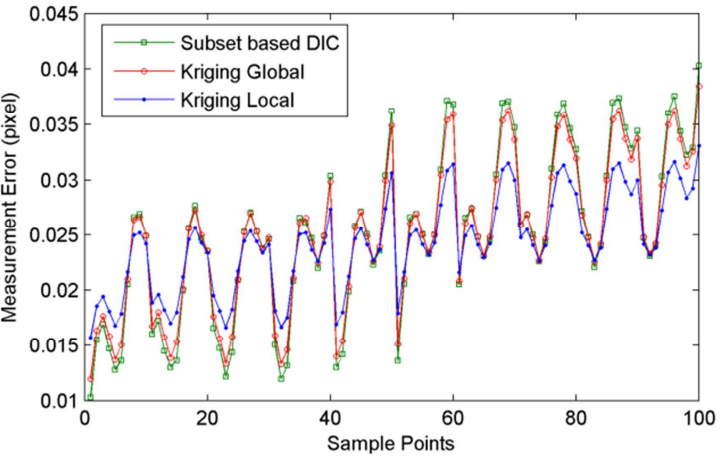

(b) y-direction

Figure 3 Numerical case study 1: residual error comparison for an affine deformation $u_{x}=0.005, u_{y}=0.005, v_{x}=0.005, v_{y}=0.005$ pixels - equation (19).

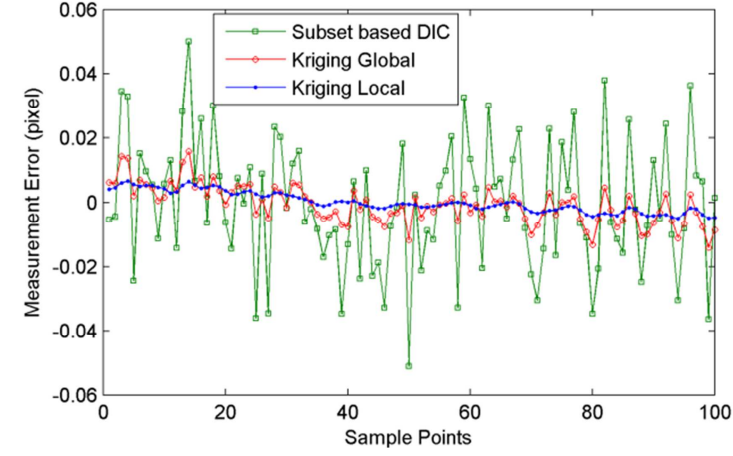

(a) $\mathrm{x}$-direction

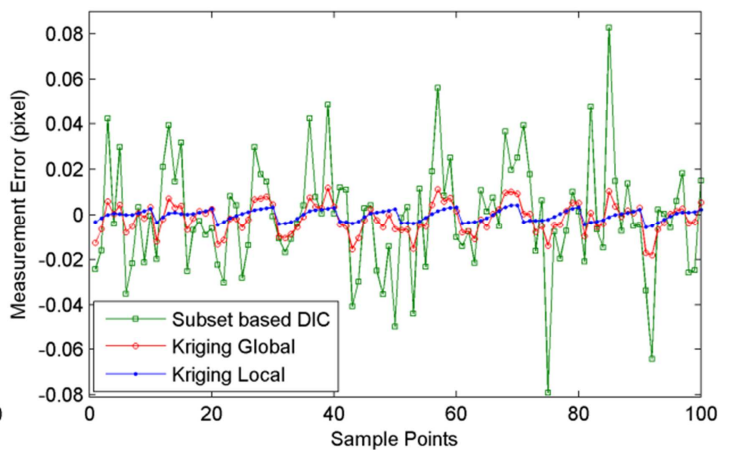

(b) y-direction

Figure 4 Numerical case study 1: residual error comparison for Gaussian image noise, zero mean, $\sigma=5$.

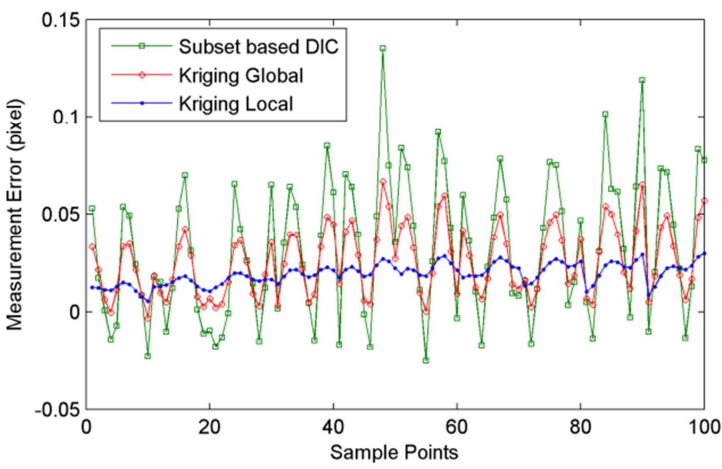

(a) x-direction

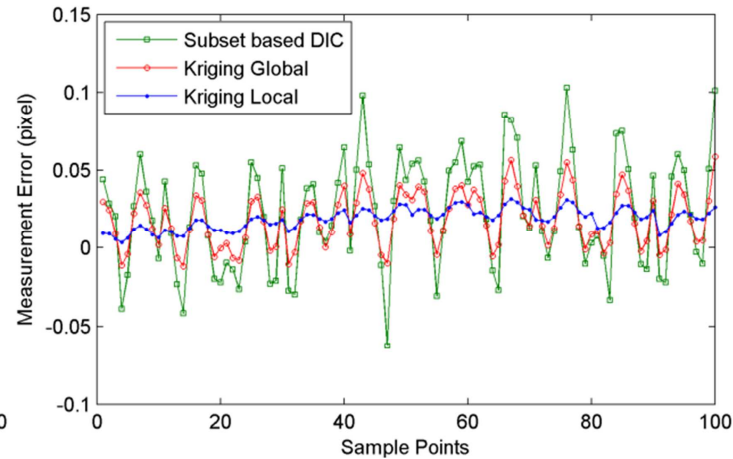

(b) y-direction

Figure 5 Numerical case study 1: residual error comparison for the combination of translation $u_{0}=0.2, v_{0}=0.3$ pixels, affine deformation $u_{x}=0.005, u_{y}=0.005, v_{x}=0.005, v_{y}=0.005$ pixels and Gaussian image noise, zero mean, $\sigma=5$.

4.2 Numerical case study 2: verification of the Kriging method for strain measurement. 
A uniaxial tensile deformation was applied to investigate the performance of proposed Kriging method in strain measurement. The pre-assigned homogenous strain was $5000 \mu \varepsilon$ in the $\mathrm{x}$ direction i.e. $u_{x}=0.005, u_{y}=0, v_{x}=0, v_{y}=0$. The same numerically generated Gaussian speckles as in Case Study 1 were used. Gaussian image noise with zero mean and $\sigma=3$ was added to the numerical images to simulate a practical noise condition. A central-area uniform grid $33 \times 33$ with a grid spacing of 13 pixels was superimposed upon the simulated image of $500 \times 500$ pixels. The displacement at the 1089 grid points was computed by the subset-based DIC using a Newton-Raphson scheme and a first-order Taylor-expansion shape function using subsets of $41 \times 41$ pixels.

Figure 6 (a) shows the displacement field calculated by the subset-based DIC where small fluctuations due to the Gaussian image noise can be observed. The regularized displacement field obtained by the Kriging local method is demonstrated in Figure 6 (b) where the error in the displacement field, due to Gaussian noise, has been significantly reduced. The strain results calculated by the PLS algorithm for different methods are illustrated in Figure 7. For this simple example, it is shown that based on the same size of strain calculation window, the Kriging local method is able to achieve superior strain results especially in the vicinity of the boundaries. The Kriging global and local methods were not significantly affected by the deficiency of valid data points at the boundaries of the strain window, since the displacement noise had already been substantially removed by the Kriging method. The boundary effect could also be reduced by extending of displacement field outside the calculation area boundaries [31], but might not be reliable in the case of complex deformations when additional errors might be introduced inadvertently.

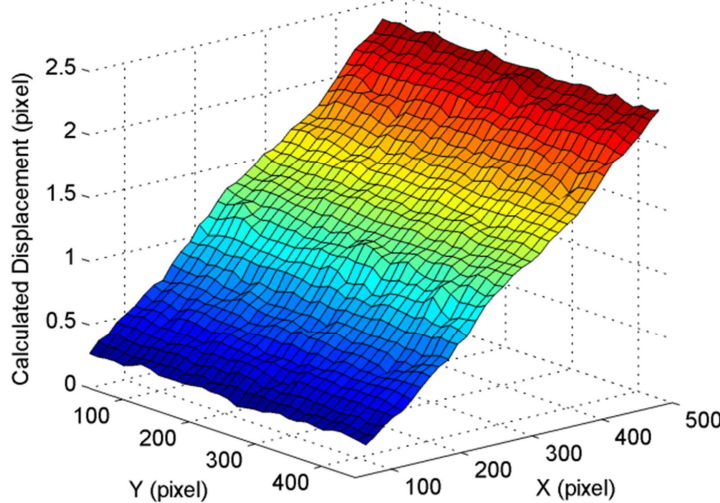

(a)

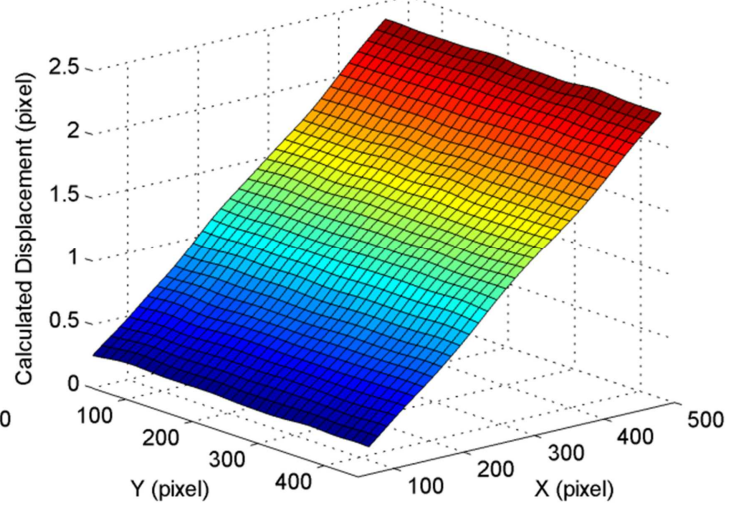

(b)

Figure 6 Numerical case study 2: calculated displacement fields, (a) by subset-based DIC using Newton-Raphson scheme, (b) by Kriging regression with local error estimate 


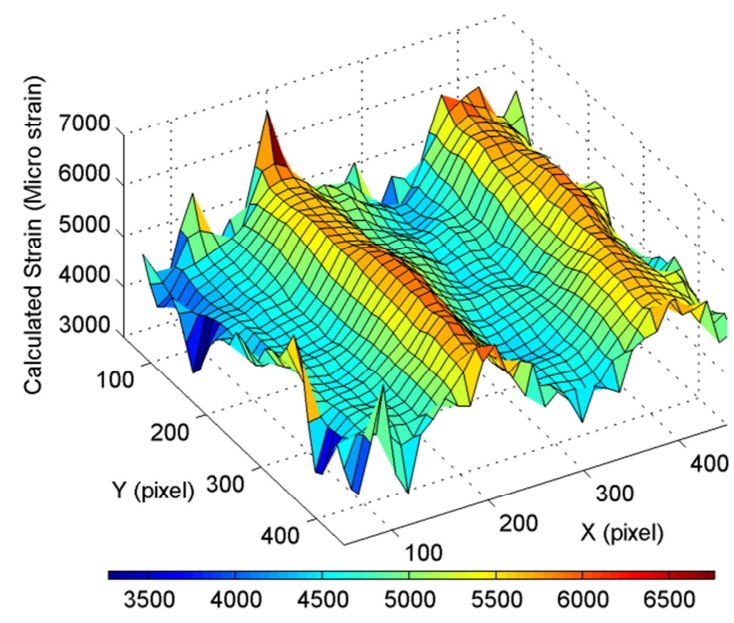

(a)

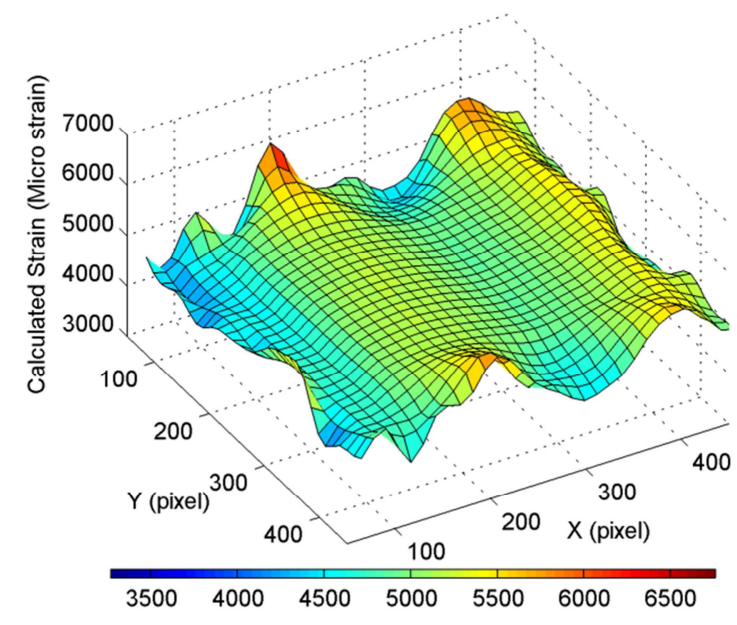

(c)

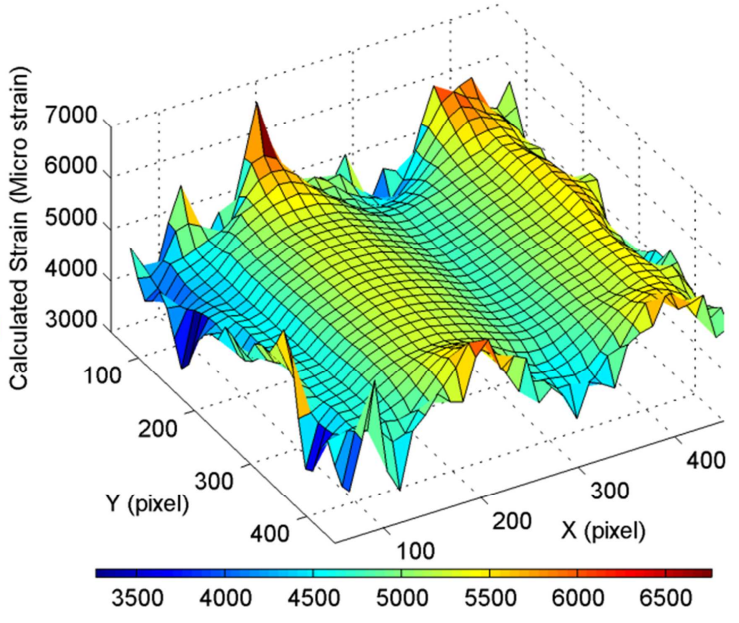

(b)

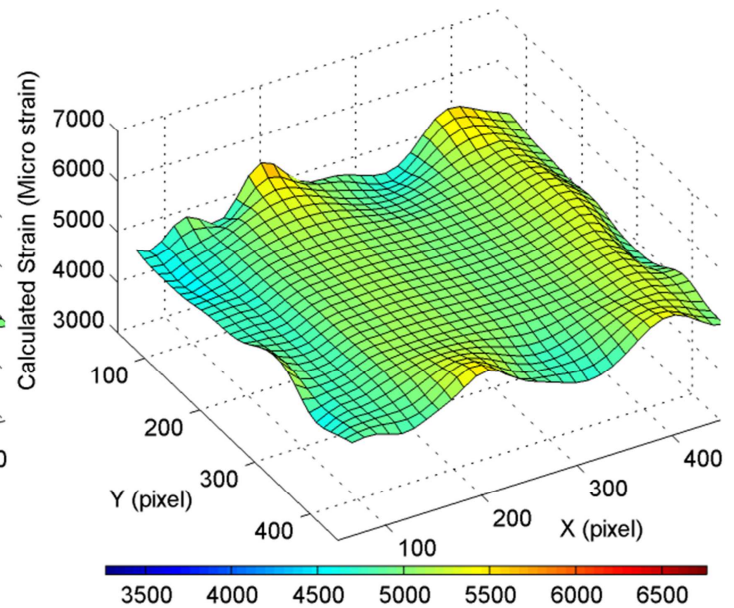

(d)

Figure 7 Numerical case study 2: calculated strain fields, (a) by subset-based DIC using $7 \times 7$ strain window, (b) by subset-based DIC using $15 \times 15$ strain window (c) by Kriging global method using $15 \times 15$ strain window (d) by Kriging local method using $15 \times 15$ strain window.

\subsection{Experimental case study: cantilever beam test with $U Q$}

The proposed Kriging regression technique was validated by using experimental data from an aluminium cantilever beam of dimensions $160 \mathrm{~mm} \times 40 \mathrm{~mm} \times 4 \mathrm{~mm}$ thick. A thin coat of quick-drying white paint (Matt Super White 1107, Plasti-kote, UK) was sprayed onto the surfaces of the cantilever beam using an aerosol can, on top of which speckles were sprayed using black paint (Matt Super Black 1102, Plastikote, UK). The beam was securely clamped to an optical table as shown in Figure 8 and, in order to avoid errors caused by relative movements, the DIC system was also clamped to the table with the camera perpendicular to the face-plane of the cantilever. Perpendicularity was checked in the present case by using a protractor, though more 
sophisticated techniques are available [36]. A vertical load, generated by a deadweight of $51 \mathrm{~kg}$ was applied at the tip, also shown in Figure 8. Two experiments (Test 1 and Test 2) were carried out using two different cantilever beams. The illumination intensity was slightly higher in Test 2 than in Test 1 as shown in Figure 9 while the speckles used in two tests were applied at different times and by different operatives. The CCD camera (Allied, Model F-125B/C) has a resolution of 1292 pixels $\times 964$ pixels with a Schneider Xenoplan lens of f-number 1.4 and $12 \mathrm{~mm}$ focal length. This combination provides resultant magnifications of 7.298 pixels $/ \mathrm{mm}$ for Test 1 and 7.326 pixels $/ \mathrm{mm}$ for Test 2 . The average speckle radius in both Test 1 and 2 was estimated to be 5 pixels. The experimental setup is also described in detail in [37].

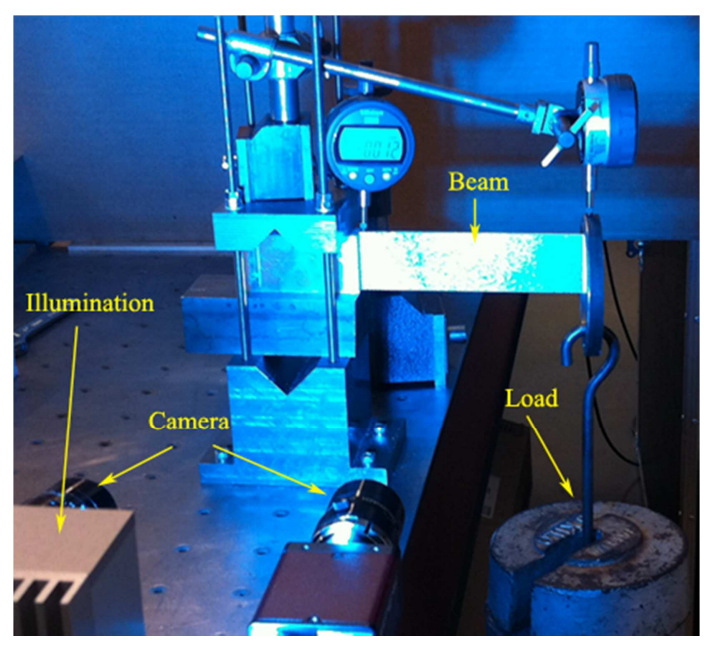

Figure 8 Experimental setup

A uniform grid of sample points $(16 \times 64)$ was selected as indicated by the red ' + ' signs shown in the reference images of Figure 9. The subset-based DIC method based on a Newton-Raphson scheme and a shape function in the form of a second-order Taylor expansion (subset size: $41 \times 41$ pixels, grid spacing: 15 pixels) was then employed to calculate the displacements of the subset centre nodes while the PLS algorithm was applied to calculate the strain results. Analytical displacement and strain results were calculated according to [38] and shown in Figure 10. The diagonal elements of the optimised correlation matrix $\mathbf{R}$ may be used to indicate the relative magnitude of quantified DIC measurement error, which differs from subset to subset. The fractional part of the diagonal element represents the extent to which the regularized sample point deviates from the original sample point. It is seen in Figure 11 that the measurement error generally increases towards to free end of the cantilever. The diagonal element of correlation matrix $\mathbf{R}$ (same for each subset) by using the Kriging global method is presented in Table 1. 


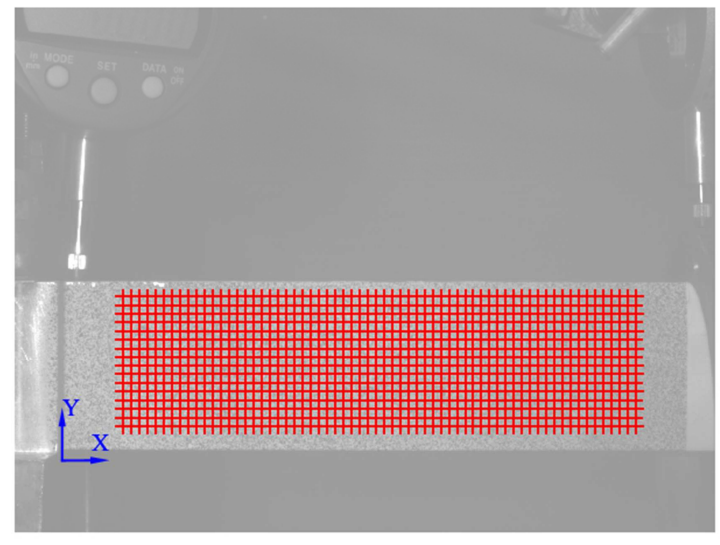

(a)

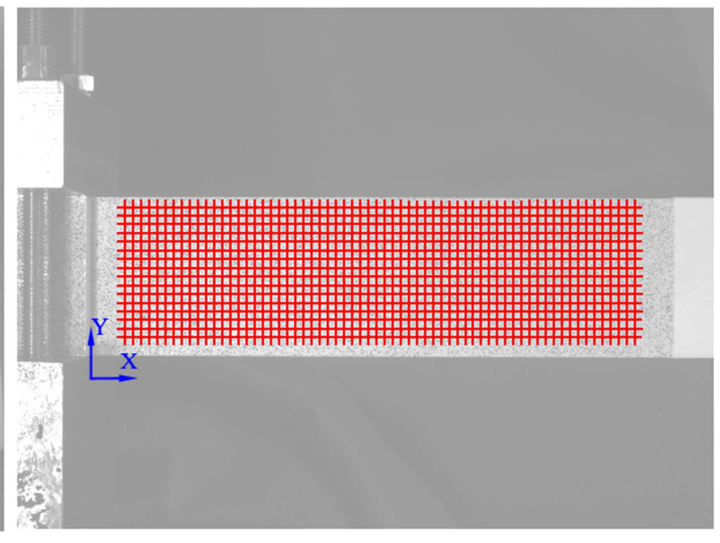

(b)

Figure 9 Distribution of sample points $(16 \times 64)$ in the reference image of the cantilever beam for Test 1 (a) and Test 2 (b)

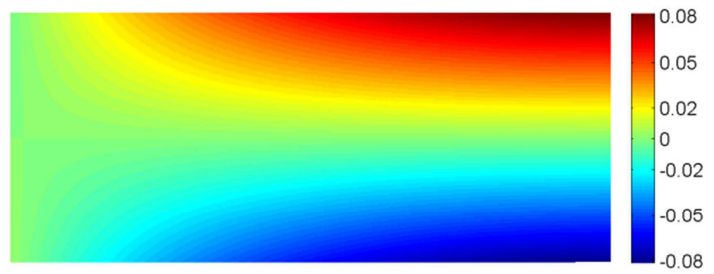

(a)

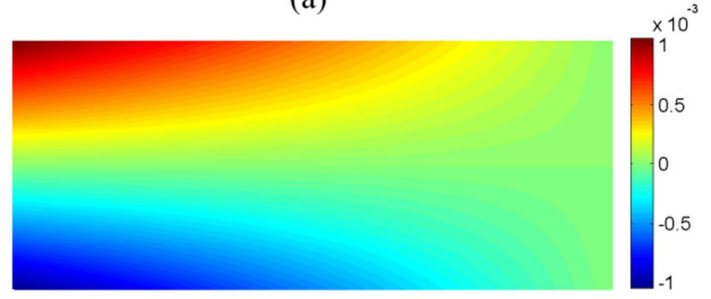

(c)

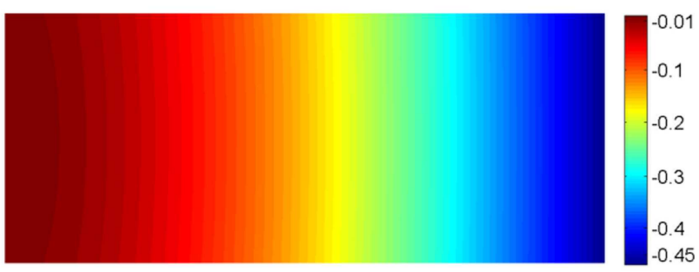

(b)

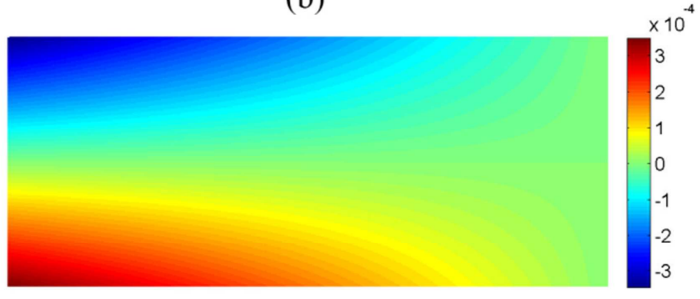

(d)

Figure 10 Analytical displacement fields (mm): (a) $x$ - and (b) $y$-directions and strain distributions: (c) $x-x$ and (d) $y-y$ strains.

Table 1 Optimized diagonal elements with global error estimate

\begin{tabular}{|c|c|}
\hline Test 1 & Test 2 \\
\hline 1.0035 & 1.0092 \\
\hline
\end{tabular}

Figure 12 shows the RMSE on the $y$-direction mean Kriging estimate. Similar results were found for the x-direction, thought the displacements are of course greatest in the $y$-direction. The tiny error is an indicator of very significant confidence in the estimate. The increase in the RMSE at the boundaries is inherent to the Kriging method. The Gaussian process deals well with interpolation (in the sense of predicting values within the convex hull of the training runs), but, as with most other meta models, it 
suffers with extrapolation. The reason is because there is no information outside the bounds so the covariance function does not have a way to interpret the relationship between the outermost point and the nearest neighbours.

For reasons of simplicity and to avoid an excessive number of figures, only the $x-x$ strain results for different methods applied to both the specimens are shown for comparison in Figure 13 and 14, i.e. (1) subset-based DIC using $21 \times 21$ strain window, (2) Kriging global method using $21 \times 21$ strain window, (3) Kriging local method using $21 \times 21$ strain window and (4) Kriging local method using the gradients from Kriging model, calculated from the Jacobian of the vector of regression functions $\mathbf{c}\left(\mathbf{x}_{p}\right)$ and the vector of correlation functions $\mathbf{r}\left(\mathbf{x}_{p}\right)$ [28]. It is worth noting that the strain fields shown in Figure 13 and 14 are linearly interpolated from the original discrete strain data $(16 \times 64)$ only for the purpose of visualisation. In order to quantify the similarity between post-processing results and analytical results, an image decomposition technique based on Tchebichef polynomials [39, 40] was used to represent each dataset and the concordance correlation coefficient [41] employed to compare the resultant moments. Specifically, 400 Tchebichef moments were used and the corresponding concordance correlation coefficients are listed in Table 2 where it is seen that Kriging regression with error estimation shows superior correlation with the analytical solution than does the subset-based DIC method. From the results in Figures 13 and 14 and Table 2, it can be seen that superior results are achieved using Kriging regression with local error estimation, reducing the difference between the estimated strain field and the analytical solution. The DIC measurement error is reduced by post-processing with local Kriging regression.

Table 2 Concordance correlation coefficient based on Tchebichef image decomposition for the strain fields in $x-x$

\begin{tabular}{|c|c|c|}
\hline & Test 1 & Test 2 \\
\hline $\begin{array}{c}\text { Subset-based DIC } \\
\text { PLS 21×21 strain window }\end{array}$ & 0.9131 & 0.9327 \\
\hline $\begin{array}{c}\text { Kriging global method } \\
\text { PLS 21×21 strain window }\end{array}$ & 0.9579 & 0.9520 \\
\hline $\begin{array}{c}\text { Kriging local method } \\
\text { PLS 21×21 strain window }\end{array}$ & 0.9733 & 0.9783 \\
\hline $\begin{array}{c}\text { Kriging local method } \\
\text { using Kriging gradients }\end{array}$ & 0.9703 & 0.9795 \\
\hline
\end{tabular}

In order to quantify the uncertainty on strain results, a multivariate Gaussian sampling technique [42] described in Section 3.4 can be applied based on the Kriging mean values and covariance matrix [28] to generate a series of random samples of the displacement field. For reasons of reducing the computational cost, 3 local regions 
were selected on the cantilever beam labelled A, B and C in Figure 15. Each region contains $6 \times 6$ sample point and a further 1030 uniformly distributed new untried points. 10000 displacement fields were generated and the corresponding strain fields calculated by the finite difference method which was also used to compute the gradients of the densely sampled displacement field. Finally the strain field uncertainty in the local region was determined in terms of the standard deviation.

Figure 16 illustrates the standard deviation of the strain results of the 3 chosen local regions. In Figure 17 the estimated probability densities and the corresponding 95\% confidence intervals of the strains are illustrated at 3 chosen points (labelled a, b and c, one each in the 3 local regions as shown in Figure 15). It is found that the greatest uncertainty appears at point $\mathrm{c}$, close to the loading point. The strain at point $\mathrm{b}$ at midspan and on the neutral axis is the most confidently predicted.

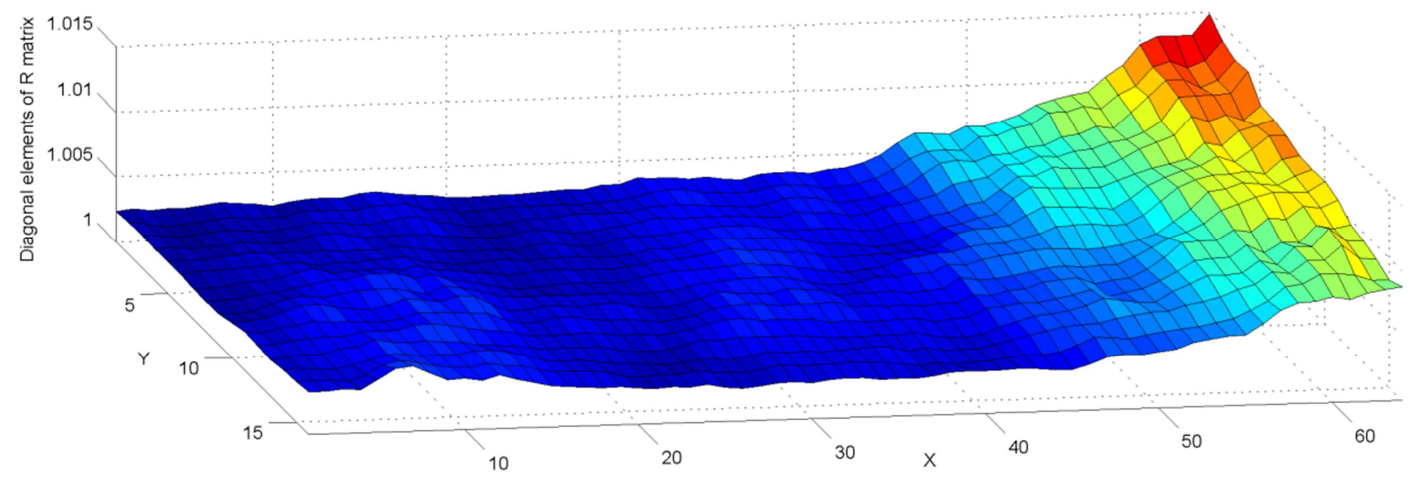

(a) Test 1

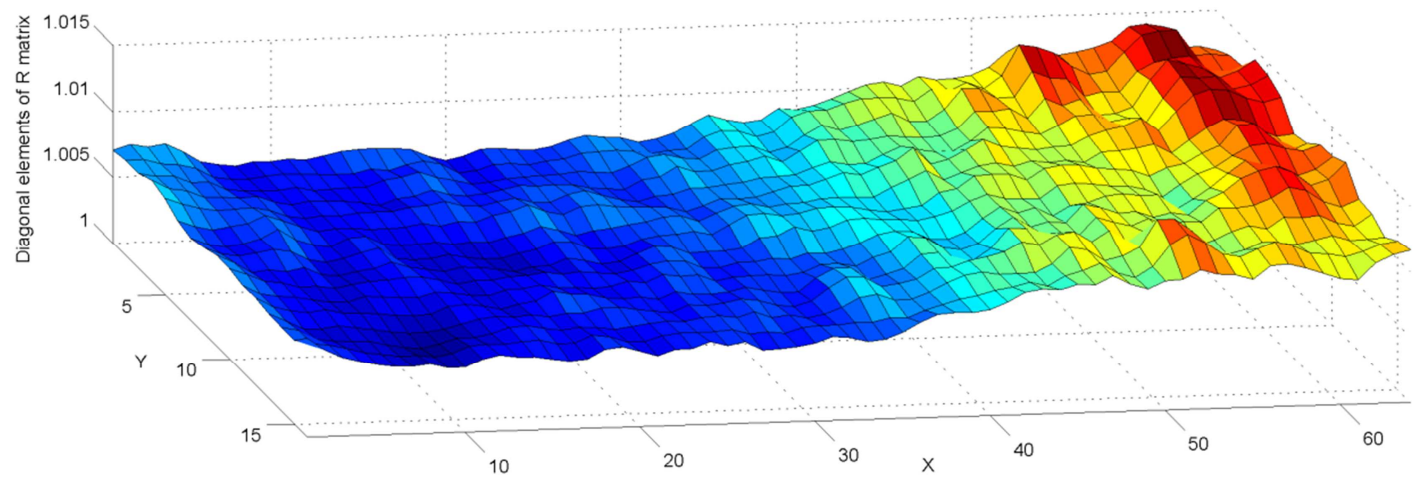

(b) Test 2

Figure 11 Diagonal elements of the optimized R matrix (16×64 centre nodes) in (a) Test 1 , (b) Test 2 


\section{Discussion}

As shown in Figure 13 and 14, applying the proposed local Kriging regression method to the displacement data obtained by classical subset-based DIC significantly improves the accuracy of the estimated displacement and strain fields. Also, the Gaussian process, which forms part of the Kriging model, allows for UQ on estimated displacement and strain fields. There is, however, a penalty to be paid for such improvements, in terms of computational cost. This might be reduced by using the two fast algorithms, FMLE and FSV proposed in [29], to accelerate the optimisation process in the Kriging local method without loss of accuracy.

For the second numerical case study (constant strain and Gaussian noise), the calculated strain results based on the Kriging gradients were found to be better than the strain results based on local-fitting gradients when a small strain calculation window, smaller than $9 \times 9$, was chosen, but slightly worse for large strain calculation windows greater than $9 \times 9$. There was found to be small, but not very significant, differences observed in the strain results calculated by Kriging gradients and locally fitted gradients for the experimental cantilever-beam case study (Figures 13(c) and (d) and 14(c) and (d)). With the regularized Kriging local method the displacement and strain results were shown to be improved in all the case studies.

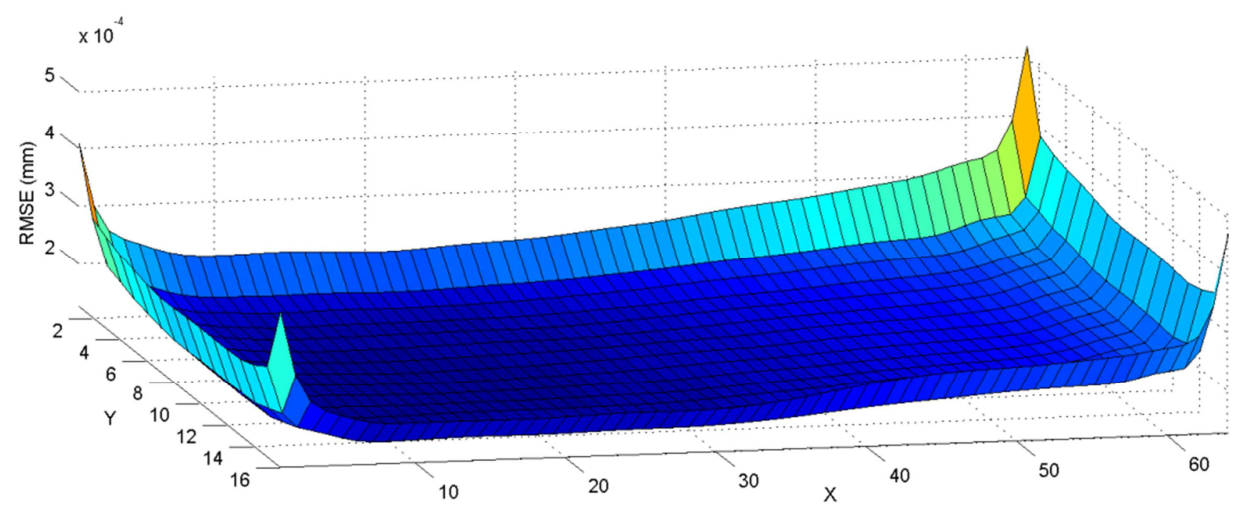

(a) Test 1

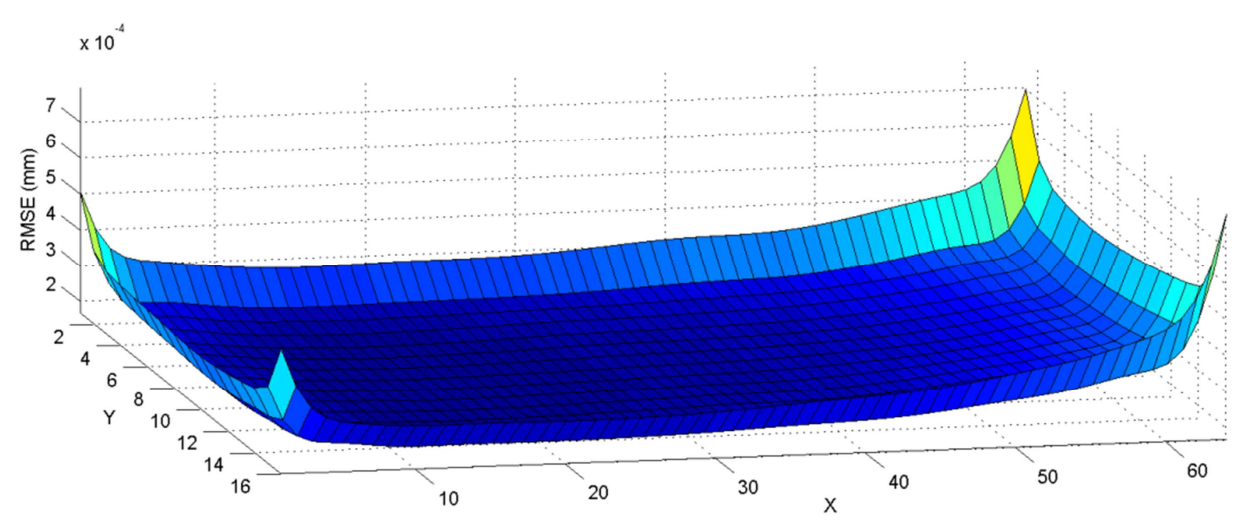

(b) Test 2 
Figure 12 RMSE on the mean Kriging estimate of the displacement field ( $y$-direction): (a) Test 1; (b) Test 2.

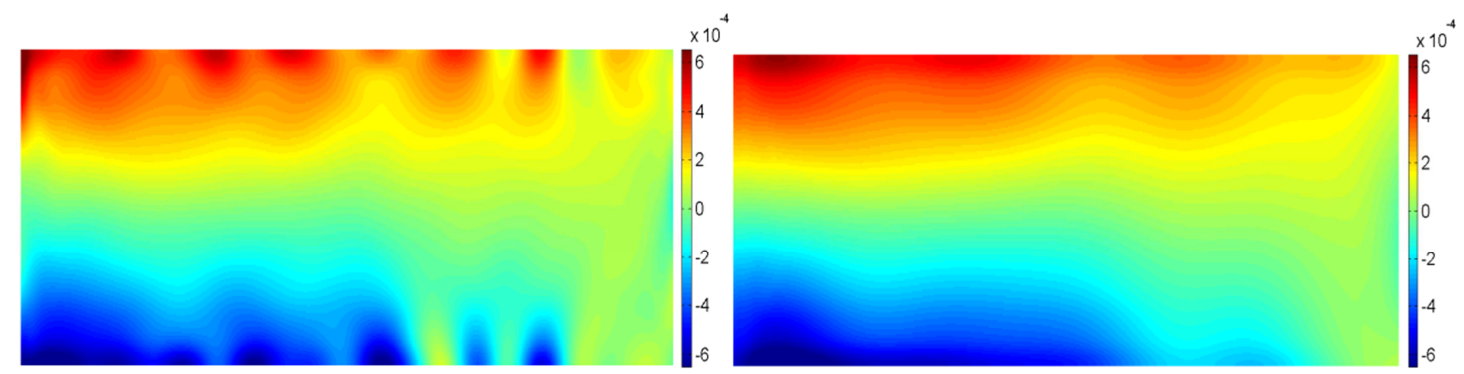

(a)

(b)

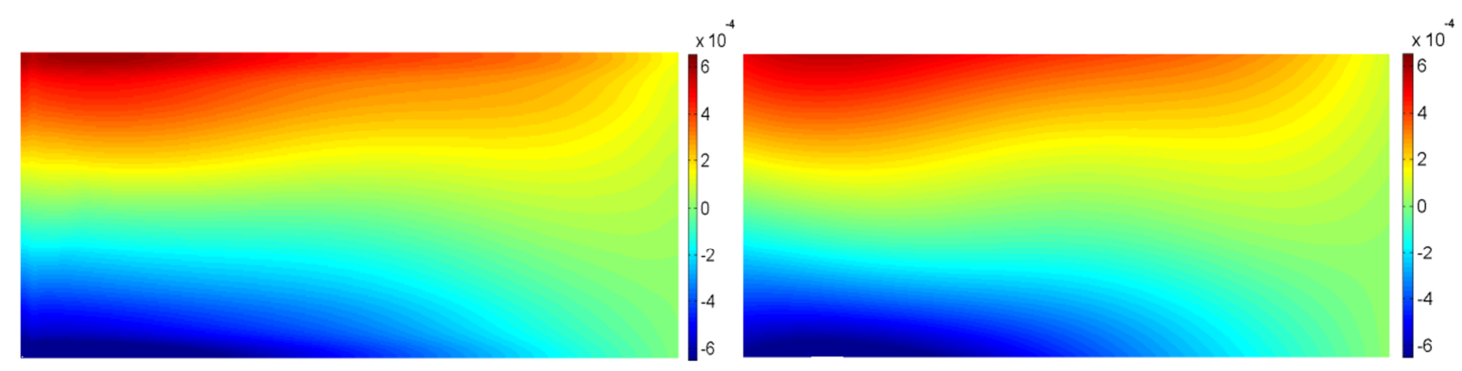

(c)

(d)

Figure 13 Test $1 x$-x strain field: (a) subset-based DIC using 21×21 strain window; (b) Kriging global method using $21 \times 21$ strain window; (c) Kriging local method using $21 \times 21$ strain window. (d) Kriging local method using Kriging gradients

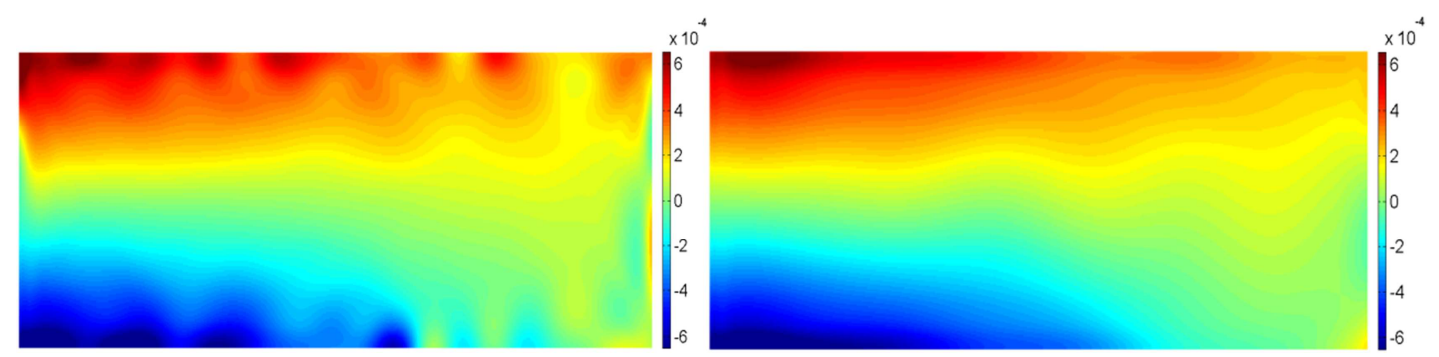

(a)

(b)

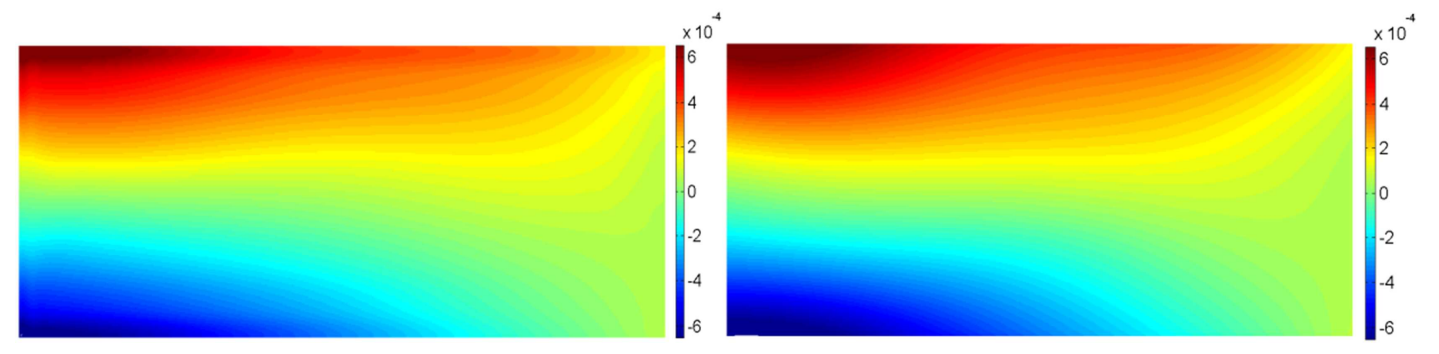

(c)

(d) 
Figure 14 Test $2 x-x$ strain field: (a) subset-based DIC using $21 \times 21$ strain window; (b) Kriging global method using $21 \times 21$ strain window; (c) Kriging local method using $21 \times 21$ strain window. (d) Kriging local method using Kriging gradients

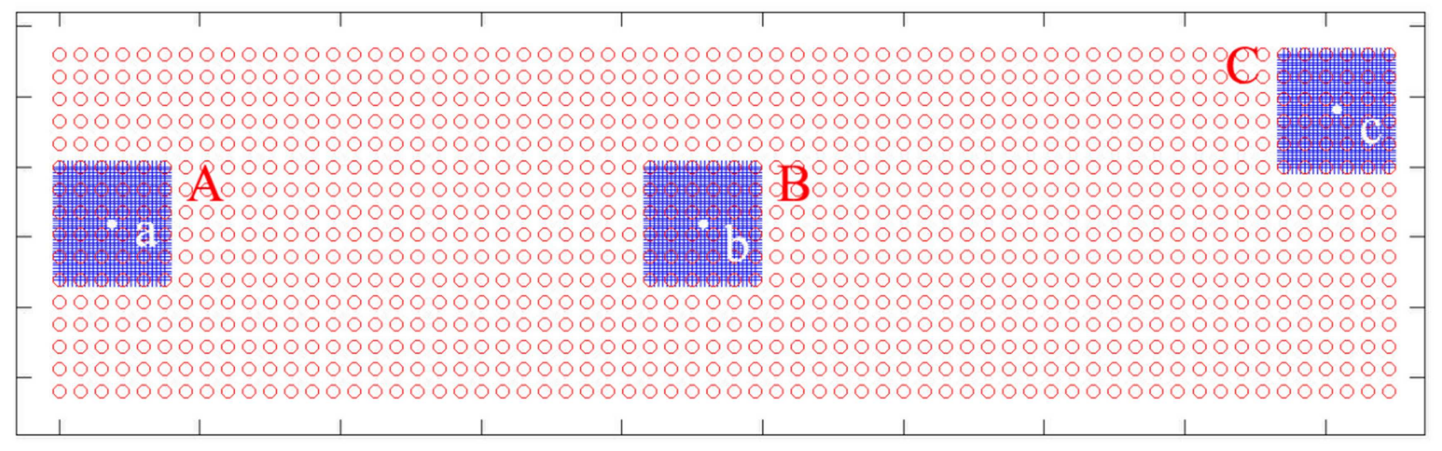

Figure 153 local regions (A, B and C) are chosen on the beam in Test 2; each region contains $6 \times 6$ sample points shown as red 'o' markers and other 1030 uniformly distributed new predicted points shown as blue ' + ' markers; $a, b$ and $c$ are the points chosen from the same location of the 3 regions

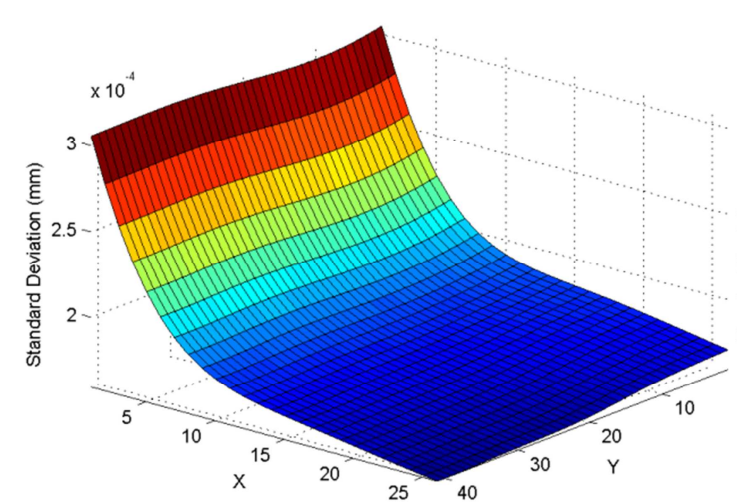

(a)

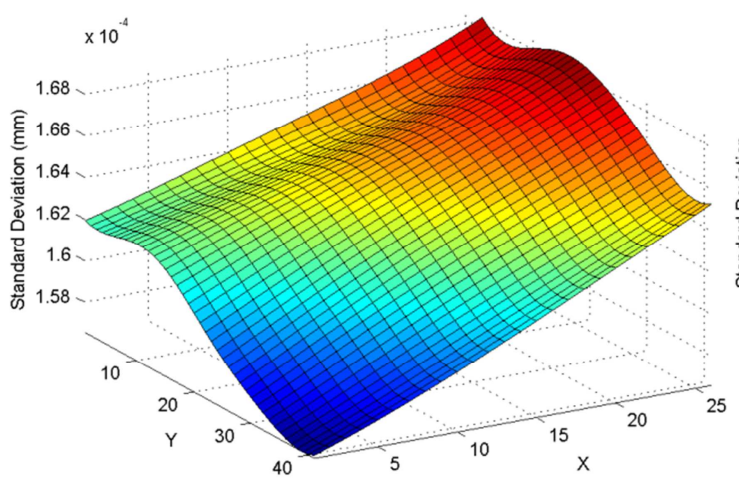

(c)

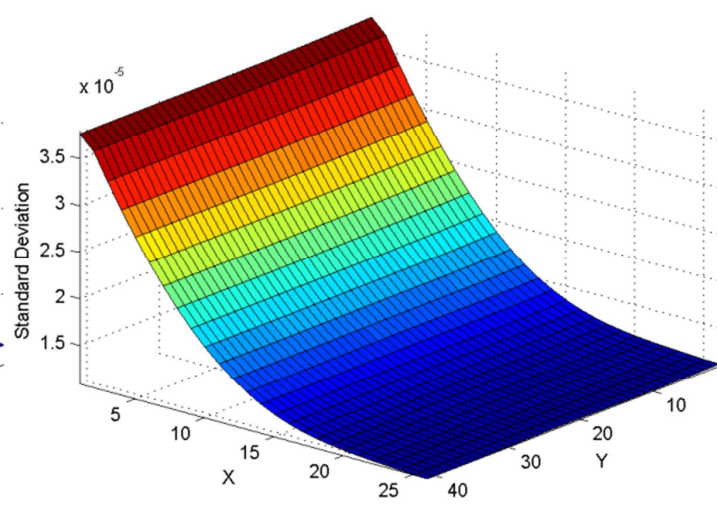

(b)

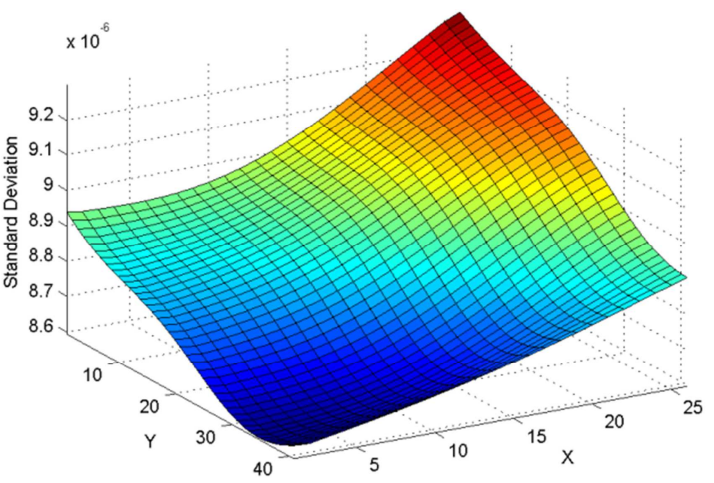

(d) 


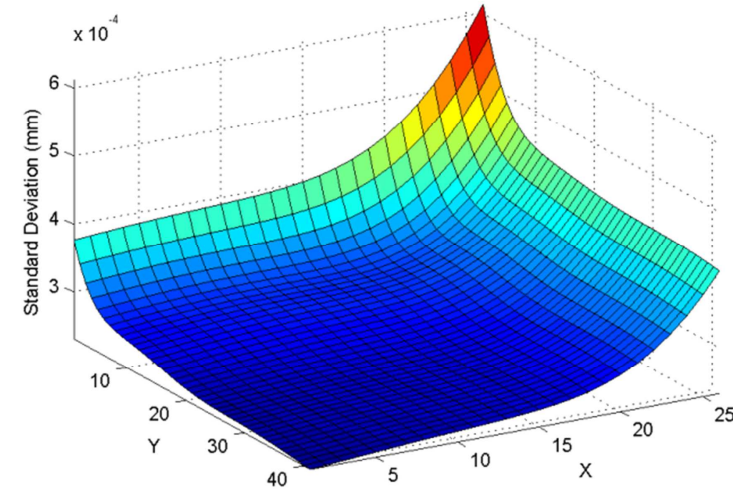

(e)

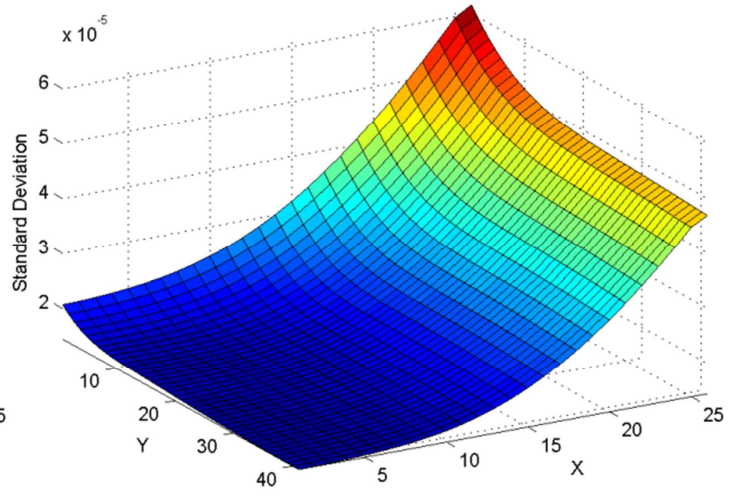

(f)

Figure 16 Displacement STD ( $x$ direction) shown in (a), (c) and (e) and Strain STD ( $x$ - $x$ direction) shown in (b), (d) and (f) based on 10000 random samples of the local displacement fields, from top to bottom: Region A, B and C

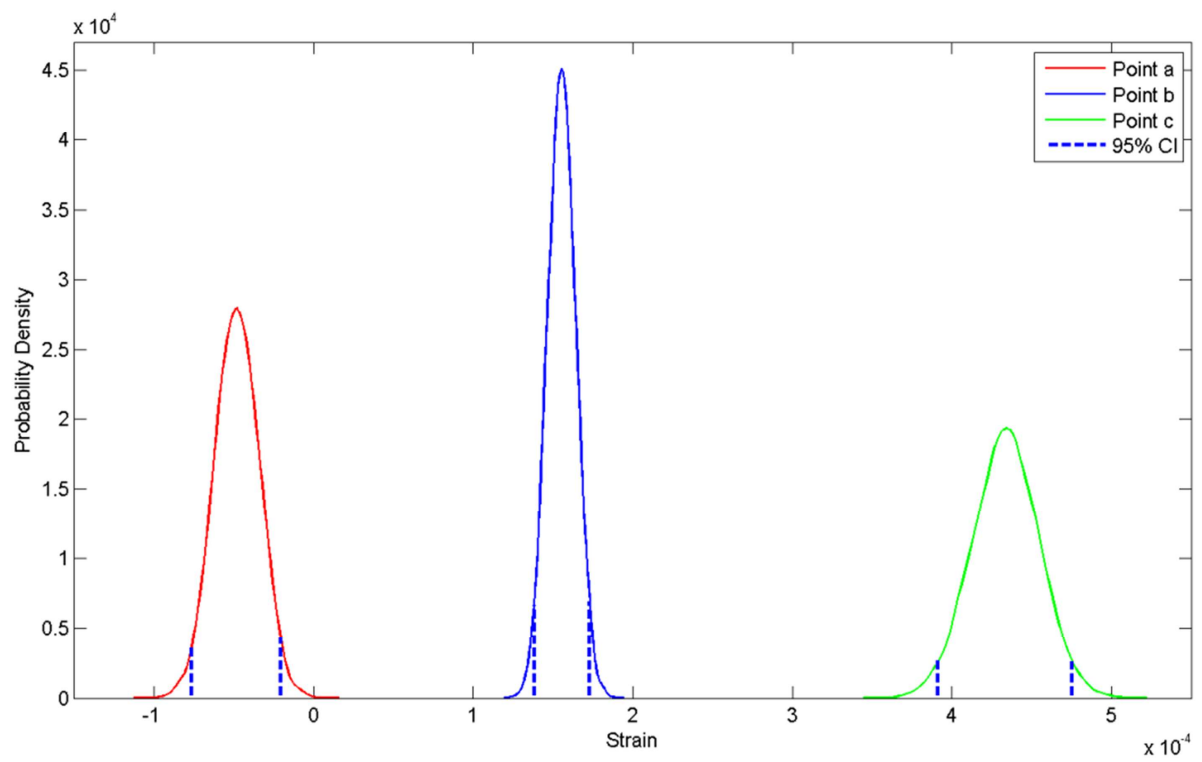

Figure 17 The probability density for the strains and 95\% confidence interval of the 3 chosen points in the 3 regions respectively (as shown in Figure 15)

\section{Conclusion}

A subset-by-subset approximation of DIC measurement error is derived and introduced into the leading-diagonal terms of the Kriging correlation matrix. This leads to a Kriging regression with local error estimation based on diagonal elements of the inverse Hessian matrix and the SSD residual, for the post-processing of measured data produced by subset-based DIC. Unlike spline or other interpolation methods, the proposed approach not only allows for regression of the model upon the measured data, but also incorporates a Gaussian process that enables RMSEs and standard deviations to be determined on the estimated displacement and strain fields. The methodology is supported by both numerical and experimental case studies. All 
the case studies show that the proposed Kriging local method out-performs the Kriging global method and is able to improve the accuracy of measured subset-based DIC data and achieve more accurate strain results.

\section{Appendix 1}

In this study the displacements uncertainty $\boldsymbol{\varepsilon}_{e}\left(\mathbf{x}_{s}\right)$ at an arbitrarily chosen pixel with coordinates $\left(x_{s}, y_{s}\right)$ is derived using a second-order shape function model,

$$
\boldsymbol{\varepsilon}_{e}\left(\mathbf{x}_{s}\right)=\boldsymbol{\eta}^{\mathrm{T}}\left(\mathbf{x}_{s}\right) \boldsymbol{\rho}_{e}
$$

where $\boldsymbol{\eta}\left(\mathbf{x}_{s}\right)$ denotes the shape function coefficients,

$$
\boldsymbol{\eta}\left(\mathbf{x}_{s}\right)=\left[\begin{array}{llllll}
1 & \Delta x_{s} & \Delta y_{s} & \frac{1}{2}\left(\Delta x_{s}\right)^{2} & \frac{1}{2}\left(\Delta y_{s}\right)^{2} & \Delta x_{s} \Delta y_{s}
\end{array}\right]^{\mathrm{T}}
$$

and $\boldsymbol{\rho}_{e}$ is the matrix of the uncertainty of variables in the two directions $x, y$,

$$
\boldsymbol{\rho}_{e}=\left[\begin{array}{ll}
\boldsymbol{\rho}_{u} & \boldsymbol{\rho}_{v}
\end{array}\right]=\left[\begin{array}{llllll}
\mathrm{u}_{0} & \mathrm{u}_{x} & \mathrm{u}_{y} & \mathrm{u}_{x x} & \mathrm{u}_{y y} & \mathrm{u}_{x y} \\
v_{0} & v_{x} & v_{y} & v_{x x} & v_{y y} & v_{x y}
\end{array}\right]^{\mathrm{T}}
$$

where $\Delta x_{s}$ and $\Delta y_{s}$ are the distances between the pixel point $\mathbf{x}_{s}$ and the centre node of the subset at $\mathbf{x}_{c}$.

The form of local error estimate is developed according to the most commonly used DIC criterion $[3,43]$ i.e. the SSD,

$$
C_{S S D}=\sum_{s=1}^{N \times N}\left[g\left(\tilde{\mathbf{x}}_{s}\right)-f\left(\mathbf{x}_{s}\right)\right]^{2}
$$

or, by equations (1), (2), (3) and (23),

$$
C_{S S D}=\sum_{s=1}^{N \times N}\left(g\left(\boldsymbol{\tau}_{s}+\boldsymbol{\eta}^{\mathrm{T}}\left(\mathbf{x}_{s}\right) \boldsymbol{\rho}_{e}\right)+\zeta_{g}\left(\boldsymbol{\tau}_{s}+\boldsymbol{\eta}^{\mathrm{T}}\left(\mathbf{x}_{s}\right) \boldsymbol{\rho}_{e}\right)-\left(f\left(\mathbf{x}_{s}\right)+\zeta_{f}\left(\mathbf{x}_{s}\right)\right)\right)^{2}
$$

Alternatives to the SSD criterion include the normalized sum of squared differences (NSSD), zero-normalized sum of squared differences (ZNSSD) etc. The Cross Correlation (CC), which is related to and can also be deduced from the SSD criterion $[3,43]$, may also be used.

\section{The Hessian matrix and determination of $\boldsymbol{p}_{e}$ :}

In equation (24) the coordinate uncertainty $\boldsymbol{\eta}^{\mathrm{T}}\left(\mathbf{x}_{s}\right) \boldsymbol{\rho}_{e}$ may be considered to be small, in which case the first-order Taylor expansion leads to, 


$$
\begin{aligned}
\mathrm{C}_{S S D}\left(\boldsymbol{\rho}_{e}\right) & =\sum_{s=1}^{N \times N}\left(g\left(\boldsymbol{\tau}_{s}\right)+\nabla g\left(\boldsymbol{\tau}_{s}\right) \cdot \boldsymbol{\eta}^{\mathrm{T}}\left(\mathbf{x}_{s}\right) \boldsymbol{\rho}_{e}+\zeta_{g}\left(\boldsymbol{\tau}_{s}\right)\right. \\
& \left.+\nabla \zeta_{g}\left(\boldsymbol{\tau}_{s}\right) \cdot \boldsymbol{\eta}^{\mathrm{T}}\left(\mathbf{x}_{s}\right) \boldsymbol{\rho}_{e}-f\left(\mathbf{x}_{s}\right)-\zeta_{f}\left(\mathbf{x}_{s}\right)\right)^{2}
\end{aligned}
$$

where $\nabla g\left(\boldsymbol{\tau}_{s}\right)$ represents the grey gradient. It is known that $g\left(\boldsymbol{\tau}_{s}\right)=f\left(\mathbf{x}_{s}\right)$ and

$$
\nabla g\left(\boldsymbol{\tau}_{s}\right)+\nabla \zeta_{g}\left(\boldsymbol{\tau}_{s}\right)=\nabla\left(g\left(\boldsymbol{\tau}_{s}\right)+\zeta_{g}\left(\boldsymbol{\tau}_{s}\right)\right)=\nabla \bar{g}\left(\boldsymbol{\tau}_{s}\right),
$$

so that equation (25) may be simplified as,

$$
\mathrm{C}_{S S D}\left(\boldsymbol{\rho}_{e}\right)=\sum_{s=1}^{N \times N}\left(\nabla \bar{g}\left(\boldsymbol{\tau}_{s}\right) \cdot \boldsymbol{\eta}^{\mathrm{T}}\left(\mathbf{x}_{s}\right) \boldsymbol{\rho}_{e}+\zeta_{g}\left(\boldsymbol{\tau}_{s}\right)-\zeta_{f}\left(\mathbf{x}_{s}\right)\right)^{2}
$$

If the gradient terms in $x$ and $y$ directions are separated out, then

$$
\begin{gathered}
\mathrm{C}_{S S D}\left(\boldsymbol{\rho}_{e}\right)=\sum_{s=1}^{N \times N}\left(\nabla \bar{g}_{x}\left(\boldsymbol{\tau}_{s}\right) \cdot \boldsymbol{\eta}^{\mathrm{T}}\left(\mathbf{x}_{s}\right) \boldsymbol{\rho}_{u}+\nabla \bar{g}_{y}\left(\boldsymbol{\tau}_{s}\right) \cdot \boldsymbol{\eta}^{\mathrm{T}}\left(\mathbf{x}_{s}\right) \boldsymbol{\rho}_{v}\right. \\
\left.+\zeta_{g}\left(\boldsymbol{\tau}_{s}\right)-\zeta_{f}\left(\mathbf{x}_{s}\right)\right)^{2}
\end{gathered}
$$

Minimisation of the SSD requires that, $d C_{S S D} / d \boldsymbol{\rho}_{u}=d C_{S S D} / d \boldsymbol{\rho}_{v}=0$ which leads to the following expression for the determination of $\left(\begin{array}{ll}\boldsymbol{\rho}_{u}^{\mathrm{T}} & \boldsymbol{\rho}_{v}^{\mathrm{T}}\end{array}\right)$,

$$
\mathbf{H}\left(\begin{array}{l}
\boldsymbol{\rho}_{u} \\
\boldsymbol{\rho}_{v}
\end{array}\right)=\mathbf{b}
$$

where,

$$
\mathbf{H}=\left[\begin{array}{cccccc}
\sum\left(\nabla \bar{g}_{x} \cdot \eta_{1}\right)^{2} & \cdots & \sum\left(\nabla \bar{g}_{x}\right)^{2} \eta_{1} \eta_{6} & \sum\left(\nabla \bar{g}_{y} \cdot \eta_{1}\right)\left(\nabla \bar{g}_{x} \cdot \eta_{1}\right) & \cdots & \sum\left(\nabla \bar{g}_{y} \cdot \eta_{6}\right)\left(\nabla \bar{g}_{x} \cdot \eta_{1}\right) \\
\vdots & & \vdots & \vdots & & \vdots \\
\sum\left(\nabla \bar{g}_{x}\right)^{2} \eta_{1} \eta_{6} & \cdots & \sum\left(\nabla \bar{g}_{x} \cdot \eta_{6}\right)^{2} & \sum\left(\nabla \bar{g}_{y} \cdot \eta_{1}\right)\left(\nabla \bar{g}_{x} \cdot \eta_{6}\right) & \cdots & \sum\left(\nabla \bar{g}_{y} \cdot \eta_{6}\right)\left(\nabla \bar{g}_{x} \cdot \eta_{6}\right) \\
\sum\left(\nabla \bar{g}_{x} \cdot \eta_{1}\right)\left(\nabla \bar{g}_{y} \cdot \eta_{1}\right) & \cdots & \sum\left(\nabla \bar{g}_{x} \cdot \eta_{6}\right)\left(\nabla \bar{g}_{y} \cdot \eta_{1}\right) & \sum\left(\nabla \bar{g}_{y} \cdot \eta_{1}\right)^{2} & \cdots & \sum\left(\nabla \bar{g}_{y}\right)^{2} \eta_{1} \eta_{6} \\
\vdots & & \vdots & \vdots & & \vdots \\
\sum\left(\nabla \bar{g}_{x} \cdot \eta_{1}\right)\left(\nabla \bar{g}_{y} \cdot \eta_{6}\right) & \cdots & \sum\left(\nabla \bar{g}_{x} \cdot \eta_{6}\right)\left(\nabla \bar{g}_{y} \cdot \eta_{6}\right) & \sum\left(\nabla \bar{g}_{y}\right)^{2} \eta_{1} \eta_{6} & \cdots & \sum\left(\nabla \bar{g}_{y} \cdot \eta_{6}\right)^{2}
\end{array}\right]
$$

is an approximation to the Hessian matrix when the second-order partial derivatives are considered to be negligible [1,44]. The terms $\nabla \bar{g}_{x}\left(\boldsymbol{\tau}_{s}\right), \nabla \bar{g}_{y}\left(\boldsymbol{\tau}_{s}\right)$ and $\eta_{i}\left(\mathbf{x}_{s}\right)$ are abbreviated to $\nabla \bar{g}_{x}, \nabla \bar{g}_{y}$ and $\eta_{i}$. The vector $\mathbf{b}$ in equation (28) may be written as, 


$$
\mathbf{b}=-\left(\begin{array}{c}
\sum_{s=1}^{N \times N}\left(\nabla \bar{g}_{x}\left(\boldsymbol{\tau}_{s}\right) \eta_{1}\left(\mathbf{x}_{s}\right)\right) \cdot\left(\zeta_{g}\left(\boldsymbol{\tau}_{s}\right)-\zeta_{f}\left(\mathbf{x}_{s}\right)\right) \\
\vdots \\
\sum_{s=1}^{N \times N}\left(\nabla \bar{g}_{x}\left(\boldsymbol{\tau}_{s}\right) \eta_{6}\left(\mathbf{x}_{s}\right)\right) \cdot\left(\zeta_{g}\left(\boldsymbol{\tau}_{s}\right)-\zeta_{f}\left(\mathbf{x}_{s}\right)\right) \\
\sum_{s=1}^{N \times N}\left(\nabla \bar{g}_{y}\left(\boldsymbol{\tau}_{s}\right) \eta_{1}\left(\mathbf{x}_{s}\right)\right) \cdot\left(\zeta_{g}\left(\boldsymbol{\tau}_{s}\right)-\zeta_{f}\left(\mathbf{x}_{s}\right)\right) \\
\vdots \\
\sum_{s=1}^{N \times N}\left(\nabla \bar{g}_{y}\left(\boldsymbol{\tau}_{s}\right) \eta_{6}\left(\mathbf{x}_{s}\right)\right) \cdot\left(\zeta_{g}\left(\boldsymbol{\tau}_{s}\right)-\zeta_{f}\left(\mathbf{x}_{s}\right)\right)
\end{array}\right)
$$

The vector of uncertainties is then given by,

$$
\left(\begin{array}{l}
\boldsymbol{\rho}_{u} \\
\boldsymbol{\rho}_{v}
\end{array}\right)=\mathbf{H}^{-1} \mathbf{b}
$$

\section{Estimation of variance}

The grey-level uncertainty was defined as independent and identically distributed, $\mathcal{N}\left(0, \sigma_{\zeta}^{2}\right)$, in Section 2 , at each pixel. Therefore, by linearisation,

$$
\operatorname{Cov}\left(\begin{array}{l}
\boldsymbol{\rho}_{u} \\
\boldsymbol{\rho}_{v}
\end{array}\right) \cong \sigma_{\zeta}^{2} \cdot \mathbf{J} \cdot \mathbf{J}^{T}
$$

where $\mathbf{J}$ is the Jacobian matrix, which from equation (31) is given by

$$
\mathbf{J}=\mathbf{H}^{-1} \mathbf{b}^{\prime}
$$

where,

$$
\mathbf{b}^{\prime}=\left[\begin{array}{llllll}
\frac{\partial \mathbf{b}}{\partial \zeta_{g}\left(\boldsymbol{\tau}_{1}\right)} & \cdots & \frac{\partial \mathbf{b}}{\partial \zeta_{g}\left(\boldsymbol{\tau}_{N \times N}\right)} & \frac{\partial \mathbf{b}}{\partial \zeta_{f}\left(\boldsymbol{\tau}_{1}\right)} & \cdots & \frac{\partial \mathbf{b}}{\partial \zeta_{f}\left(\boldsymbol{\tau}_{N \times N}\right)}
\end{array}\right]
$$

and,

$$
\mathbf{b}^{\prime}\left(\mathbf{b}^{\prime}\right)^{\mathrm{T}}=2 \mathbf{H}
$$

Finally, from equations (32), (33) and (35) it is found that,

$$
\operatorname{Cov}\left(\begin{array}{l}
\boldsymbol{\rho}_{u} \\
\boldsymbol{\rho}_{v}
\end{array}\right) \cong 2 \sigma_{\zeta}^{2} \cdot \mathbf{H}^{-1}
$$

The uncertainty at the centre node of the subset is the first term in each row of $\boldsymbol{\rho}_{e}$ as defined in equation (22). The displacement variance at the centre node in one subset may then be approximated $[1,15]$ as, 


$$
\left\{\begin{array}{l}
\operatorname{Var}\left(u_{0}\right) \cong 2 \sigma_{\zeta}^{2} \cdot\left(\mathbf{H}^{-1}\right)_{11} \\
\operatorname{Var}\left(v_{0}\right) \cong 2 \sigma_{\zeta}^{2} \cdot\left(\mathbf{H}^{-1}\right)_{77}
\end{array}\right.
$$

The derivations above is limited by the assumption of Gaussian uncertainty and therefore provides only a lower-bound error estimate. A more general case may be derived from the residual of the SSD criterion $[17,18]$ such that the $2 \sigma_{\zeta}^{2}$ in equation (37) is replaced by,

$$
\frac{C_{S S D}}{N^{2}}=\frac{\sum_{s=1}^{N \times N}\left[g\left(\tilde{\mathbf{x}}_{s}\right)-f\left(\mathbf{x}_{s}\right)\right]^{2}}{N \times N}
$$

Then the local uncertainty terms $m_{x j}$ and $m_{y j}$ associated with $x$ - and $y$-direction displacements for the $j^{\text {th }}$ subset in equation (14) are given by,

$$
\begin{aligned}
& m_{x j}=\frac{C_{S S D j}}{N^{2}} \cdot\left(\mathbf{H}^{-1}\right)_{11} \\
& m_{y j}=\frac{C_{S S D j}}{N^{2}} \cdot\left(\mathbf{H}^{-1}\right)_{77}
\end{aligned}
$$

\section{Acknowledgement}

The first author wishes to acknowledge the support of the Chinese Scholarship Council (CSC) and the University of Liverpool. The experimental work was supported by funding from the European Community's Seventh Framework Programme under Grant Agreement no NMP-SA-2012-319116. EAP is a recipient of a Royal Society Wolfson Research Merit Award.

\section{References}

[1] Sutton MA, Orteu J-J, Schreier HW. Image correlation for shape, motion and deformation measurements : basic concepts, theory and applications. New York, N.Y.: Springer; 2009.

[2] Zhao JQ, Zeng P, Pan B, Lei LP, Du HF, He WB, et al. Improved Hermite finite element smoothing method for full-field strain measurement over arbitrary region of interest in digital image correlation. Optics and Lasers in Engineering 2012; 50(11): 1662-1671.

[3] Pan B, Qian KM, Xie HM, Asundi A. Two-dimensional digital image correlation for inplane displacement and strain measurement: a review. Measurement Science \& Technology 2009; 20(6).

[4] Pan B, Asundi A, Xie HM, Gao JX. Digital image correlation using iterative least squares and pointwise least squares for displacement field and strain field measurements. Optics and Lasers in Engineering 2009; 47(7-8): 865-874.

[5] Pan B. Bias error reduction of digital image correlation using Gaussian pre-filtering. Optics and Lasers in Engineering 2013; 51(10): 1161-1167. 
[6] Banerjee KS, Carr RN. Ridge Regression - Biased Estimation for Non-Orthogonal Problems. Technometrics 1971; 13(4): 895-\&.

[7] Forrester AlJ, Keane AJ, Bressloff NW. Design and analysis of "Noisy" computer experiments. Aiaa Journal 2006; 44(10): 2331-2339.

[8] de Baar JHS, Percin M, Dwight RP, van Oudheusden BW, Bijl H. Kriging regression of PIV data using a local error estimate. Experiments in Fluids 2014; 55(1).

[9] Jones DR. A taxonomy of global optimization methods based on response surfaces. Journal of Global Optimization 2001; 21(4): 345-383.

[10] Matheron G. Principles of geostatistics. Econimic Geology 1963; 58: 1246-1266.

[11] Cressie N. The Origins of Kriging. Mathematical Geology 1990; 22(3): 239-252.

[12] Cressie N. Statistics for spatial data. Rev. ed. Wiley series in probability and mathematical statistics Applied probability and statistics. New York: Wiley; 1993.

[13] Wikle CK, Berliner LM. A Bayesian tutorial for data assimilation. Physica D-Nonlinear Phenomena 2007; 230(1-2): 1-16.

[14] Charonko JJ, Vlachos PP. Estimation of uncertainty bounds for individual particle image velocimetry measurements from cross-correlation peak ratio. Measurement Science \& Technology 2013; 24(6).

[15] Wang YQ, Sutton MA, Bruck HA, Schreier HW. Quantitative Error Assessment in Pattern Matching: Effects of Intensity Pattern Noise, Interpolation, Strain and Image Contrast on Motion Measurements. Strain 2009; 45(2): 160-178.

[16] Pan B, Lu ZX, Xie HM. Mean intensity gradient: An effective global parameter for quality assessment of the speckle patterns used in digital image correlation. Optics and Lasers in Engineering 2010; 48(4): 469-477.

[17] Ke XD, Schreier HW, Sutton MA, Wang YQ. Error Assessment in Stereo-based Deformation Measurements Part II: Experimental Validation of Uncertainty and Bias Estimates. Experimental Mechanics 2011; 51(4): 423-441.

[18] Wieneke B, Prevost R. DIC Uncertainty Estimation from Statistical Analysis of Correlation Values. Advancement of Optical Methods in Experimental Mechanics 2014.

[19] Wang DZ, DiazDelaO FA, Wang WZ, Mottershead JE. Full-field digital image correlation with Kriging regression. Optics and Lasers in Engineering 2015; 67: 105-115.

[20] Tikhonov AN. Numerical methods for the solution of ill-posed problems. Mathematics and its applications. Dordrecht ; Boston: Kluwer Academic Publishers; 1995.

[21] Forrester AIJ, Sóbester AS, Keane AJ. Engineering design via surrogate modelling : a practical guide. Chichester, West Sussex, England ; Hoboken, NJ: J. Wiley; 2008.

[22] Phillip RL, Sutton MA, Wang Y, Miller TJ. Uncertainty quantification for digital image correlation. in Proceedings of the SEM Annual Conference. 2009: Albuquerque New Mexico USA.

[23] Zhou YH, Sun C, Song YT, Chen JB. Image pre-filtering for measurement error reduction in digital image correlation. Optics and Lasers in Engineering 2015; 65: 46-56.

[24] Mazzoleni P, Matta F, Zappa E, Sutton MA, Cigada A. Gaussian pre-filtering for uncertainty minimization in digital image correlation using numerically-designed speckle patterns. Optics and Lasers in Engineering 2015; 66: 19-33.

[25] Hengl T, Heuvelink GBM, Rossiter DG. About regression-kriging: From equations to case studies. Computers \& Geosciences 2007; 33(10): 1301-1315.

[26] Sacks J, Welch WJ, Mitchell TJ, Wynn HP. Design and Analysis of Computer Experiments. Statistical Science, 1989; 4(4): 409-435.

[27] Hengl T, Heuvelink GBM, Stein A. A generic framework for spatial prediction of soil variables based on regression-kriging. Geoderma 2004; 120(1-2): 75-93.

[28] Lophaven SN, Nielsen HB. DACE A Matlab Kriging Toolbox. Denmark, Editor. 2002. 
[29] de Baar JHS, Dwight RP, Bijl H. Speeding up Kriging through fast estimation of the hyperparameters in the frequency-domain. Computers \& Geosciences 2013; 54: 99106.

[30] Ranjan P, Haynes R, Karsten R. A Computationally Stable Approach to Gaussian Process Interpolation of Deterministic Computer Simulation Data. Technometrics 2011; 53(4): 366-378.

[31] Wattrisse B, Chrysochoos A, Muracciole JM, Nemoz-Gaillard M. Analysis of strain localization during tensile tests by digital image correlation. Experimental Mechanics 2001; 41(1): 29-39.

[32] Thomas DB, Luk W. Sampling from the Multivariate Gaussian distribution using reconfigurable hardware. Fccm 2007: 15th Annual leee Symposium on FieldProgrammable Custom Computing Machines, Proceedings 2007: 3-12.

[33] Rasmussen CE, Williams CKI. Gaussian processes for machine learning. Adaptive computation and machine learning. Cambridge, Mass.: MIT Press; 2006.

[34] Zhou P, Goodson KE. Subpixel displacement and deformation gradient measurement using digital image/speckle correlation (DISC). Optical Engineering 2001; 40(8): 16131620.

[35] Zhou YH, Chen YQ. Propagation function for accurate initialization and efficiency enhancement of digital image correlation. Optics and Lasers in Engineering 2012; 50(12): 1789-1797.

[36] Felipe-Sese L, Siegmann P, DiazDelO FA, Patterson EA. Simultaneous in-and-out-ofplane displacement measurements using fringe projection and digital image correlation. Optics and Lasers in Engineering 2014; 52: 66-74.

[37] Hack E, Lin X, Patterson EA, Sebastian CM. A reference material for establishing uncertainties in full-field displacement measurements, Measurement Science \& Technology, revised manuscript submitted.

[38] Timoshenko S, Goodier JN. Theory of elasticity. 3d ed. Engineering societies monographs. New York,: McGraw-Hill; 1969.

[39] Wang WZ, Mottershead JE, Sebastian CM, Patterson EA. Shape features and finite element model updating from full-field strain data. International Journal of Solids and Structures 2011; 48(11-12): 1644-1657.

[40] Wang WZ, Mottershead JE. Adaptive moment descriptors for full-field strain and displacement measurements. Journal of Strain Analysis for Engineering Design 2013; 48(1): 16-35.

[41] Labeas G, Pasialis, V.P., Lin, X. \& Patterson, E.A. On the validation of solid mechanics models using optical measurements and data decomposition. Simulation Modelling Practice \& Theory 2015; 52: 92-107.

[42] Sandor Z, Andras P. Alternative sampling methods for estimating multivariate normal probabilities. Journal of Econometrics 2004; 120(2): 207-234.

[43] Tong W. An evaluation of digital image correlation criteria for strain mapping applications. Strain 2005; 41(4): 167-175.

[44] Lu H, Cary PD. Deformation measurements by digital image correlation: Implementation of a second-order displacement gradient. Experimental Mechanics 2000; 40(4): 393-400. 